\title{
DIRICHLET SERIES AND AUTOMORPHIC FORMS ON UNITARY GROUPS
}

\author{
BY
}

\author{
TOBIAS ORLOFF ${ }^{1}$
}

ABSTRACT. In a special case our unitary group takes the form

$$
G=\left\{\left.g \in \mathrm{GL}(p+2, \mathrm{C})\right|^{t} \bar{g} R g=R\right\} .
$$

Here

$$
R=\left(\begin{array}{ccc}
S & 0 & 0 \\
0 & 0 & 1 \\
0 & -1 & 0
\end{array}\right)
$$

is a skew-Hermitian matrix with entries in an imaginary quadratic number field $K$. We suppose that $-i R$ has signature $(p+1,1)$. This group acts naturally on the symmetric domain

$$
D=\left\{w \in \mathbf{C}^{p}, z \in \mathbf{C} \mid \operatorname{Im}(z)>-\frac{1}{2}^{t} \bar{w} S w\right\} .
$$

If $\Gamma=G \cap \operatorname{SL}\left(p+2, \mathcal{O}_{K}\right)$ with $\mathcal{O}_{K}$ the ring of integers in $K$, then an automorphic form $f(w, z)$ with respect to $\Gamma$ has an expansion $\sum_{r} g_{r}(w) \cdot e^{2 \pi i r z}$. The functions $g_{r}(w)$ are theta functions. Given another automorphic form $g(w, z)$ with an expansion $\sum_{s} h_{s}(w) \cdot e^{2 \pi i s z}$ we define a Dirichlet series $\sum_{r}\left\langle g_{r}, h_{r}\right\rangle r^{-s}$. Here $\left\langle g_{r}, h_{r}\right\rangle$ is a certain positive definite inner product on the space of theta functions. The series is obtained as an integral of Rankin type:

$$
\int_{P_{\Gamma} \backslash D} f \bar{g} \cdot\left(\operatorname{Im}(z)+\frac{1}{2} i^{t} \bar{w} S w\right)^{s} d w d \bar{w} d z d \bar{z}
$$

with $P_{\Gamma} \subseteq \Gamma$ a subgroup of "translations". The series is analytically continued by studying the Eisenstein series arising when the above integral is transformed into an integral over $\Gamma \backslash D$. In the case $p=1$ our results have an application to some recent work of Shintani, where the Euler product attached to an eigenfunction of the Hecke operators is obtained, up to some simple factors, as a series of the above type.

1. Introduction. The principal result of this paper is the analytic continuation of a certain type of Dirichlet series. The series are constructed by means of the Rankin convolution of automorphic forms on unitary groups. We shall restrict mainly to the case of unitary groups of signature $(2,1)$, remarking on the general case in $\$ 10$. In the case of signature $(2,1)$, a construction such as ours has appeared in a paper of Shintani, and we shall discuss his results at the end of the introduction. To describe our results, let $K$ be a totally imaginary quadratic extension of a totally real number field $F$. (Such a $K$ is called a $C M$-field.) Suppose $[F: \mathbf{Q}]=n$ and $\Delta$ is a collection of

Received by the editors November 6, 1983.

1980 Mathematics Subject Classification. Primary 10D20; Secondary 14 G10.

${ }^{1}$ Supported by NSF Grant No. MCS83-11675. 
$n$ distinct embeddings of $K$ into $\mathbf{C}$, no two of which are complex conjugates of one another. (Such a $\Delta$ is called a $C M$-type.) Suppose $S \in K$ satisfies $S^{\rho}=-S$ and $-i S^{\mu}>0$ for all $\mu \in \Delta$. Here $\rho$ denotes the nontrivial element of $\operatorname{Gal}(K / F)$, which we also denote by '-', a symbol which further denotes complex conjugation when applied to elements of $\mathbf{C}$. Let

$$
R=\left(\begin{array}{ccc}
S & 0 & 0 \\
0 & 0 & 1 \\
0 & -1 & 0
\end{array}\right) \in \mathrm{GL}(3, K)
$$

and define an algebraic group

$$
G_{\mathbf{Q}}=\left\{\left.g \in \mathrm{GL}(3, K)\right|^{t} g^{\rho} R g=R\right\} .
$$

Here $t$ denotes the transpose. We consider $G_{\mathbf{Q}}$ as the Q-rational points of a Q-rational algebraic group $G$. We can identify the real points $G_{\mathbf{R}}$ of this group with the product $\prod_{\mu \in \Delta} G_{\mu}$, where

$$
G_{\mu}=\left\{\left.g \in \mathrm{GL}(3, \mathrm{C})\right|^{t} \bar{g} R^{\mu} g=R^{\mu}\right\} .
$$

Here $R^{\mu} \in \mathrm{GL}(3, \mathrm{C})$ is the matrix obtained by embedding the components of $R$ into $C$ via $\mu$. Thus $G_{\mu}$ is a unitary group of signature $(2,1)$.

For elements $g \in G_{\mathbf{Q}}$ and $\mu \in \Delta$ we have $g^{\mu} \in G_{\mu}$, and we consider $G_{\mathbf{Q}}$ as embedded in $G_{\mathbf{R}}$ by this means. For any element $g \in G_{\mathbf{R}}$ with

$$
g=\left(\begin{array}{lll}
A & B & C \\
D & E & F \\
H & I & J
\end{array}\right)
$$

we denote by

$$
g_{\mu}=\left(\begin{array}{ccc}
A_{\mu} & B_{\mu} & C_{\mu} \\
D_{\mu} & E_{\mu} & F_{\mu} \\
H_{\mu} & I_{\mu} & J_{\mu}
\end{array}\right)
$$

the $\mu$-component of $g$. Define a Hermitian symmetric domain $D=\prod_{\mu \in \Delta} D_{\mu}$, where

$$
D_{\mu}=\left\{(w, z) \mid w \in \mathbf{C}, z \in \mathbf{C}, \operatorname{Im}(z)>-\frac{1}{2} i \bar{w} S^{\mu} w\right\} .
$$

For a point $z=(w, z) \in D$ we denote by $z_{\mu}=\left(w_{\mu}, z_{\mu}\right) \in D_{\mu}$ the $\mu$-component of $z$. In the above notation the element $g \in G_{\mathbf{R}}$ acts on $z \in D$ by the formula

$$
g(z)=\left(\frac{A w+B z+C}{H w+i Z+J}, \frac{D w+E z+F}{H w+I z+J}\right) .
$$

Here we multiply two $n$-tuples of numbers by multiplying their corresponding entries. For more details see [7].

Given a tuple $k=\left(k_{\mu}\right)_{\mu \in \Delta}$ of nonnegative integers we define a factor of automorphy

$$
j(g, z)^{k}=\prod_{\mu \in \Delta}\left(H_{\mu} w_{\mu}+I_{\mu} z_{\mu}+J_{\mu}\right)^{k_{\mu}}
$$


Then $j(g h, z)^{k}=j(g, h(z))^{k} \cdot j(h, z)^{k}$ for $g, h \in G_{\mathbf{R}}$ and $z \in D$. (In general, given tuples of numbers $x=\left(x_{\mu}\right)_{\mu \in \Delta}$ and $t=\left(t_{\mu}\right)_{\mu \in \Delta}$ we define $x^{t}=\prod_{\mu \in \Delta} x_{\mu}^{t_{\mu}}$ whenever this makes sense.) Choosing an arithmetic subgroup $\Gamma$ of $G_{\mathbf{Q}}$, say for example $\Gamma=G_{\mathbf{Q}} \cap \operatorname{SL}\left(3, \mathcal{O}_{K}\right)$, where $\mathcal{O}_{K}$ is the ring of integers in $K$, we can define an automorphic form of weight $k$ with respect to $\Gamma$ to be a holomorphic function $f$ : $D \rightarrow$ C such that

$$
f(\gamma(z))=j(\gamma, z)^{k} f(z)
$$

for all $\gamma \in \Gamma$. Although our domain is not a tube domain, such a function has a Fourier-Jacobi expansion of the form

$$
f(z)=\sum_{r \in \hat{J}} g_{r}(w) e^{2 \pi i \cdot \operatorname{tr}(r \cdot z)} .
$$

Here $\hat{J}$ is a certain lattice in $K, \operatorname{tr}(r \cdot z)=\sum_{\mu \in \Delta} r^{\mu} z_{\mu}$, and the $g_{r}(w)$ are functions of $w \in \mathbf{C}^{n}$. Moreover, we can identify a suitable subgroup $P_{\Gamma} \subseteq \Gamma$ of "translations". (See $\$ 2$ for the precise definition.) Given another automorphic form $g$ of weight $m$ and a Fourier-Jacobi expansion

$$
g(z)=\sum_{r \in \hat{J}} h_{r}(w) e^{2 \pi i \cdot \operatorname{tr}(r \cdot z)},
$$

we define

$$
D(s, f, g)=\int_{P_{\Gamma} \backslash D} f \bar{g}\left(\operatorname{Im}(z)+\frac{1}{2} i \bar{w} S w\right)^{(k+m) / 2+s+2} d \Omega .
$$

Here $s \in \mathbf{C}$ is considered an $n$-tuple $(s, \ldots, s)$ and $d \Omega$ is the $G_{\mathbf{R}}$-invariant measure on $D$. With a suitable restriction on $f$ and $g$ this integral converges for $\operatorname{Re}(s)$ sufficiently large and can be evaluated to give

$$
\begin{aligned}
D(s, f, g)= & \operatorname{vol}\left(\mathbf{C}^{n} / J\right)(4 \pi)^{-(k+m) / 2-s} \Gamma_{n}\left(\frac{1}{2}(k+m)+s\right) \\
& \cdot \sum_{r \in \hat{J} / U}\left\langle g_{r}, h_{r}\right\rangle r^{-(k+m) / 2-s} .
\end{aligned}
$$

Here $\Gamma_{n}\left(\frac{1}{2}(k+m)+s\right)=\prod_{\mu \in \Delta} \Gamma\left(\frac{1}{2}\left(k_{\mu}+m_{\mu}\right)+s\right)$ with the usual $\Gamma$-function, $J$ is a lattice associated to $\hat{J},\left\langle g_{r}, h_{r}\right\rangle$ is a certain positive definite inner product, and the sum is taken over $\hat{J}$ modulo a group of units $U$. Then our main result (Theorem 7.1) states that the function $D(s, f, g)$ possesses an analytic continuation to a meromorphic function on the whole $s$-plane. Moreover, when multiplied by a suitable product of $L$-functions and $\Gamma$-factors it becomes an entire function of $s$. Our methods do not give a functional equation in a transparent form, except in a special case (see §8).

We now discuss the paper of Shintani referred to earlier [10]. This work is concerned with automorphic forms on $S U(2,1)$. Let $f$ be such a form which is an eigenfunction of all the Hecke operators. Shintani attaches an Euler product $L(s, f)$ to $f$ and shows that $L(s, f)$ is equal, up to some simple factors, to $D\left(s, f, g_{f}\right)$ with an automorphic form $g_{f}$ dependent on $f$. Thus our main result implies the analytic continuability of $L(s, f)$. This has also been obtained by Y. Flicker by a different method [2]. 
Acknowledgement. This paper is based on the author's Ph.D. dissertation, written under the direction of Professor Goro Shimura. For his guidance and encouragement the author would like to express his sincerest thanks to Professor Shimura.

Notation. The notation of the introduction will be retained in the text. For a symmetric (or Hermitian) matrix $P$ the notation $P>0$ signifies that $P$ is positive definite. Also, for a ring $R$ with unit we denote by $R^{*}$ the group of invertible elements of $R$. For positive integers $m$ and $n$ let $R_{n}^{m}$ be the ring of $m \times n$ matrices with entries in $R$. Write $R^{m}=R_{1}^{m}$ and $R_{m}=R_{m}^{1}$. The $n \times n$ identity matrix is denoted by $1_{n}$, or simply 1 when there is no fear of confusion. We let $\operatorname{GL}(n, R)$ or $\mathrm{GL}_{n}(R)$ denote $\left(R_{n}^{n}\right)^{x}$. We use the standard symbols $\mathbf{Z}, \mathbf{Q}, \mathbf{R}$, and $\mathbf{C}$ to denote the ring of integers, rational numbers, real numbers, and complex numbers, respectively. Finally, for $z \in \mathbf{C}$ we write $e(z)=e^{2 \pi i z}$.

2. Automorphic forms and Fourier-Jacobi expansions. By a lattice $\Lambda \subseteq K_{3}$ we mean a $\mathbf{Z}$-submodule of finite, maximal $\mathbf{Z}$-rank. For such a lattice define

$$
\Gamma_{\Lambda}=\left\{\gamma \in G_{\mathbf{Q}} \mid \Lambda \gamma=\Lambda, \operatorname{det} \gamma=1\right\}
$$

Define a subgroup $N_{\mathbf{Q}}$ of $G_{\mathbf{Q}}$ by

$$
N_{\mathbf{Q}}=\left\{\gamma=\left(\begin{array}{ccc}
* & 0 & * \\
* & * & * \\
0 & 0 & *
\end{array}\right) \mid \gamma \in G_{\mathbf{Q}}\right\}
$$

Let $\Gamma$ be an arbitrary subgroup of $\Gamma_{\Lambda}$ of finite index. Let $N_{\Gamma}=N_{\mathbf{Q}} \cap \Gamma$. There is a subgroup $U$ of finite index in the group of totally positive units of $\mathcal{O}_{F}$ such that

$$
U_{\Gamma}=\left\{\left(\begin{array}{ccc}
1 & 0 & 0 \\
0 & u & 0 \\
0 & 0 & u^{-1}
\end{array}\right) \mid u \in U\right\}
$$

is a subgroup of $\Gamma$. Also there are lattices $L \subseteq K$ and $J \subseteq F$ such that $U \cdot L=L$, $U^{2} \cdot J=J$, and

$$
M(L, J) \stackrel{\operatorname{def}}{=}\left\{\left(\begin{array}{ccc}
1 & 0 & x \\
\bar{x} S & 1 & y+\frac{1}{2} \bar{x} S x \\
0 & 0 & 1
\end{array}\right) \mid x \in L, y \in J\right\}
$$

is a subgroup of $\Gamma$. Let $Z_{\Gamma}$ denote the set of elements of $\Gamma$ which act trivially on $D$. $Z_{\Gamma}$ is a finite group of matrices of the form $\zeta \cdot 1_{3}$ with a root of unity $\zeta$. Finally, let

$$
P_{\Gamma}=Z_{\Gamma} \cdot U_{\Gamma} \cdot M(L, J) .
$$

( $P_{\Gamma}$ depends on $\Gamma, U, L$ and $J$.) Then $P_{\Gamma}$ is a subgroup of finite index in $N_{\Gamma}$.

Suppose $f$ is an automorphic form of weight $k$ with respect to $\Gamma$. Let

$$
\gamma=\left(\begin{array}{lll}
1 & 0 & 0 \\
0 & 1 & y \\
0 & 0 & 1
\end{array}\right) \quad \text { with } y \in J
$$


Then from (1.5), $f(w, z+y)=f(w, z)$. Fix $w$ and consider $f$ as a function of $z$. It is then a holomorphic function on an open subset of $\mathbf{C}^{n}$ and it is invariant under translation by the lattice $J \subseteq \mathbf{R}^{n}$. (Here we are considering $F \subseteq \mathbf{R}^{n}$ via the $C M$-type $\Delta$.) It follows that $f$ has an expansion of the form

$$
f(w, z)=\sum_{r \in \hat{J}} g_{r}(w) \cdot e(\operatorname{tr}(r \cdot z)),
$$

where $\hat{J}=\left\{r \in F \mid \operatorname{tr}_{F / \mathbf{Q}}(r J) \subseteq \mathbf{Z}\right\}$ and the $g_{r}(w)$ are holomorphic functions defined everywhere on $\mathbf{C}^{n}$. We call this expression the Fourier-Jacobi expansion of $f$. Now choose

$$
\gamma=\left(\begin{array}{ccc}
1 & 0 & x \\
\bar{x} S & 1 & \frac{1}{2} \bar{x} S x \\
0 & 0 & 1
\end{array}\right) \quad \text { with } x \in L
$$

Then from (1.5), $f\left(w+x, \bar{x} S w+z+\frac{1}{2} \bar{x} S x\right)=f(w, z)$. This implies

$$
g_{r}(w+x)=g_{r}(w) e\left(-\operatorname{tr}\left(r \cdot \bar{x} S\left(w+\frac{1}{2} x\right)\right) .\right.
$$

That is, $g_{r}(w)$ is a theta function with respect to the lattice $L \subseteq \mathbf{C}^{n}$. (Here we consider $K \subseteq \mathbf{C}^{n}$ via the $C M$-type $\Delta$.) It follows that $g_{0}(w)$ is constant and $g_{r}(w)=0$ unless $r^{\mu} \geq 0$ for each $\mu \in \Delta$. Finally, choose

$$
\gamma=\left(\begin{array}{ccc}
1 & 0 & 0 \\
0 & u & 0 \\
0 & 0 & u^{-1}
\end{array}\right) \quad \text { with } u \in U
$$

Then (1.5) implies

$$
g_{r}(u w)=u^{-k} \cdot g_{r u^{2}}(w) .
$$

For an element $\gamma \in G_{\mathbf{Q}}$ define $\left.f\right|_{k} \gamma$ as the function $f(\gamma(z)) \cdot j(\gamma, z)^{-k}$. The automorphic form $f$ is called a cusp form if the constant term in the Fourier-Jacobi expansion of $\left.f\right|_{k} \gamma$ is zero for every $\gamma \in G_{\mathbf{Q}}$. It is worth noting that $f$ is necessarily a cusp form unless all the components of $k$ are equal. This follows easily from setting $r=0$ in (2.8). Also, $f$ is zero unless $\gamma^{k}=1$ for all $\gamma \in Z_{\Gamma}$.

3. Estimates for Fourier-Jacobi coefficients. Let $f(w, z)=\sum g_{r}(w) e(\operatorname{tr}(r \cdot z))$ be as in $\$ 2$, i.e. an automorphic form of weight $k$ with respect to $\Gamma$. In this section we establish estimates for the functions $f$ and $g_{r}$ which will be needed in the next section. Write

$$
q(w, z)=\operatorname{Im}(z)+\frac{1}{2} i \bar{w} S w .
$$

From formula 1.17 of [7] we have

$$
q(\gamma(z))=q(z) \cdot|j(\gamma, z)|^{-2} \text { for } \gamma \in G_{\mathbf{R}} .
$$

We now recall the main theorem of reduction theory, suitably specialized to our case (see Borel [1]). Thus, for subsets $A \subseteq \mathbf{R}^{n}, B \subseteq \mathbf{C}^{n}$, and an $n$-tuple of positive numbers $\varepsilon=\left(\varepsilon_{\mu}\right)_{\mu \in \Delta}$ define a subset $T(\varepsilon, A, B) \subseteq D$ by

$$
T(\varepsilon, A, B)=\{(w, z) \in D \mid q(w, z)>\varepsilon, \operatorname{Re}(z) \in A, w \in B\} .
$$


Here and henceforth a condition such as $x>g$ for $n$-tuples $x=\left(x_{\mu}\right)$ and $y=\left(y_{\mu}\right)$ means $x_{\mu}>y_{\mu}$ for each $\mu \in \Delta$.

Reduction theory (Theorem 1.10 of [1]). There exist compact subsets $A \subseteq \mathbf{R}^{n}$ and $B \subseteq \mathbf{C}^{n}$, an $n$-tuple of positive numbers $\varepsilon$, and a finite subset $P$ of $G_{\mathbf{Q}}$ so that

$$
D=\Gamma \bigcup_{g \in P} g(T(\varepsilon, A, B)) \text {. }
$$

Thus we can find a fundamental domain for $\Gamma$ contained in the set $\bigcup_{g \in P} g(T(\varepsilon, A, B))$.

Proposition 3.1. If $f$ is a cusp form, then the function $f(z) \cdot q(z)^{k / 2}$ is bounded on D.

Proof. Let $T(\varepsilon, A, B)$ and $P \subseteq G_{\mathrm{Q}}$ be as in the reduction theory above. By assumption $\left.f\right|_{k} \gamma$ is a cusp form for every $\gamma \in P$. It follows from the Fourier-Jacobi expansion of $\left.f\right|_{k} \gamma$ that $\left(\left.f\right|_{k} \gamma\right) \cdot q(z)^{k / 2}$ is bounded on $T(\varepsilon, A, B)$. But

$$
|f|_{k} \gamma \cdot q(z)^{k / 2}|=| f(\gamma(z)) \cdot q(\gamma(z))^{k / 2} \mid
$$

by (3.2). Therefore $\left|f(z) \cdot q(z)^{k / 2}\right|$ is bounded on $\bigcup_{\gamma \in P} \gamma T(\varepsilon, A, B)$ and hence also bounded on $D=\Gamma \cdot \cup_{\gamma \in P} \gamma T(\varepsilon, A, B)$, since $\left|f(z) \cdot q(z)^{k / 2}\right|$ is invariant under $\Gamma$.

Proposition 3.2. Let $f$ be as above.

(1) If $f$ is a cusp form,

$$
\left|g_{r}(w)\right| \leq C r^{k / 2} \cdot e\left(-\operatorname{tr}\left(r \cdot \frac{1}{2} \bar{w} S w\right)\right)
$$

for $0<r \in \hat{J}$ and with a constant $C$ independent of $r$ and $w$.

(2) If $f$ is not a cusp form,

$$
\left|g_{r}(w)\right| \leq C r^{k} \cdot e\left(-\operatorname{tr}\left(r \cdot \frac{1}{2} \bar{w} S w\right)\right)
$$

for $0<r \in \hat{J}$ and with a constant $C$ independent of $r$ and $w$.

Proof. The proof of (1) follows from the formula

$$
\operatorname{vol}\left(\mathbf{R}^{n} / J\right) \cdot g_{r}(w) e(i \cdot \operatorname{tr}(r y))=\int_{x \in \mathbf{R}^{n} / J} f(w, x+i y) e(-\operatorname{tr}(r x)) d x_{1} \cdots d x_{n}
$$

for $(w, i y) \in D$, with $y \in \mathbf{R}^{n}$. For, if $f$ is a cusp form, then by Proposition 3.1 $|f(w, x+i y)| \leq C q(w, i y)^{-k / 2}$. Substituting this in (3.6) we obtain

$$
\left|g_{r}(w)\right| \leq C q(w, i y)^{-k / 2} e(-i \cdot \operatorname{tr}(r \cdot y)) .
$$

Choosing $y=-\frac{1}{2} i \bar{w} S w+1 / r$ yields the inequality (3.4).

For the case in which $f$ is not a cusp form, we need the following lemma.

Lemma 3.3. Let $P$ be a finite subset of $G_{\mathbf{Q}}$. Given

$$
\gamma=\left(\begin{array}{lll}
* & * & * \\
* & * & * \\
a & b & c
\end{array}\right) \in \bigcup_{\alpha \in P} \gamma \Gamma \text { and } z=(w, z) \in D
$$


there is a constant $M>0$, independent of $\gamma$ and $z$, so that

$$
|a w+b z+c|^{1} \geq M \cdot \min \left(1, q(z)^{1}\right) .
$$

Here $1=(1, \ldots, 1)$.

Proof. To simplify notation let us fix $\mu \in \Delta$ and drop it as a subscript or superscript. Thus for now $a$ denotes $a_{\mu}, b$ denotes $b_{\mu}$, etc. Assume $b \neq 0$. Then

$$
|\bar{b}(a w+b z+c)| \geq|\operatorname{Im}(\bar{b} a w)+| b^{2}|\cdot \operatorname{Im}[z]+\operatorname{Im}(\bar{b} c)| .
$$

Since $\gamma \in G_{\mathbf{Q}}$ we have ${ }^{t} \bar{\gamma} R \gamma=R$. Hence

$$
\left(\begin{array}{lll}
a & b & c
\end{array}\right)\left(\begin{array}{lll}
S^{-1} & 0 & 0 \\
0 & 0 & -1 \\
0 & 1 & 0
\end{array}\right) \cdot\left(\begin{array}{l}
\bar{a} \\
\bar{b} \\
\bar{c}
\end{array}\right)=0
$$

or

$$
i a S^{-1} \cdot \bar{a}=2 \cdot \operatorname{Im}(c \bar{b}) .
$$

Using this, the right-hand side of (3.8) may be written

$$
\left.|\operatorname{Im}(\bar{b} a w)+| b\right|^{2} \cdot\left(\operatorname{Im}(z)+\frac{1}{2} i \bar{w} S w\right)-\frac{1}{2} i|b|^{2} \bar{w} S w+\frac{1}{2} i a S^{-1} \bar{a} \mid
$$

Now,

$$
\operatorname{Im}(\bar{b} a w) \geq-|\bar{b}| \cdot|a w|=-|b| \cdot\left|a \cdot i S^{-1}(-i S w)\right|
$$

Therefore

$$
\begin{aligned}
& -\frac{1}{2} i|b|^{2} \cdot \bar{w} S w+\operatorname{Im}(\bar{b} a w)+\frac{1}{2} i a S^{-1} \cdot \bar{a} \\
& \quad \geq \frac{1}{2}\left[\left(i a S^{-1} \cdot \bar{a}\right)^{1 / 2}+|b|(-i \bar{w} S w)^{1 / 2}\right]^{2} \geq 0 .
\end{aligned}
$$

Using this in (3.11) and (3.8) we obtain

$$
|\bar{b}(a w+b z+c)| \geq|b|^{2} \cdot\left(\operatorname{Im}(z)+\frac{1}{2} i \bar{w} S w\right)=|b|^{2} \cdot q(z) .
$$

This inequality should be read with the subscript (or superscript) $\mu$ in place. Since $b \neq 0$ we obtain

$$
|a w+b z+c|^{1} \geq M q(z)^{1},
$$

where $M^{2}$ is the minimum nonzero absolute value of the norm of any. element of any matrix in $\bigcup_{\alpha \in P} \alpha \Gamma$. (This exists since $\bigcup_{\alpha \in P} \alpha \Gamma \subseteq\left(1 / N \cdot \mathcal{O}_{K}\right)_{3}^{3}$ for some integer $N$.)

If $b=0$ the equation (3.10) implies $a=0$. Therefore

$$
|a w+b z+c|^{1}=|c|^{1} \geq M .
$$

This combined with (3.15) completes the proof of the lemma.

Returning to the proof of Proposition 3.2, suppose $f$ is not a cusp form. It follows from the remark at the end of $\S 2$ that $k_{\mu}=k_{\psi}$ for any two $\mu, \psi \in \Delta$. Let $z \in D$. Choose $P$ and $T(\varepsilon, A, B)$ as in the reduction theory above. It follows that there are $\alpha \in P$ and $\gamma \in \Gamma$ so that

$$
\alpha^{-1} \gamma(z) \in T(\varepsilon, A, B) \text {. }
$$


Let $z^{\prime}=\alpha^{-1} \gamma(z)$. Note that any automorphic form (cusp form or not) is bounded on $T(\varepsilon, A, B)$. Let us say $|f|_{k} \alpha \mid \leq L$ on $T(\varepsilon, A, B)$ for each $\alpha \in P$. Then

$$
\begin{aligned}
|f(z)| & =\left|f\left(\gamma^{-1} \alpha\left(z^{\prime}\right)\right)\right|=\left|f\left(\alpha\left(z^{\prime}\right)\right)\right| \cdot\left|j\left(\gamma^{-1}, \alpha\left(z^{\prime}\right)\right)\right|^{k} \\
& =|f|_{k} \alpha\left(z^{\prime}\right) \cdot j\left(\alpha^{-1} \gamma, z\right)^{-k} \mid \leq L / M^{k} \max \left(1, q(z)^{-k}\right)
\end{aligned}
$$

by Lemma 3.3. Substituting this inequality into (3.6) we obtain

$$
\left|g_{r}(w)\right| \leq e(-i \cdot \operatorname{tr}(r y)) \cdot L / M \max \left(1, q(z)^{-k}\right) .
$$

Again choose $y=-\frac{1}{2} i \bar{w} S w+1 / r$. Since $0<r \in \hat{J}$ and all the entries of $k$ are equal, it follows that $r^{k}$ is bounded below by a positive constant independent of $r$. Then

$$
\begin{aligned}
\left|g_{r}(w)\right| & \leq e\left(-\operatorname{tr}\left(r \cdot \frac{1}{2} \bar{w} S w\right)\right) \cdot e^{2 \pi n} \cdot(L / M) \max \left(1, r^{k}\right) \\
& \leq C r^{k} \cdot e\left(-\operatorname{tr}\left(r \cdot \frac{1}{2} \bar{w} S w\right)\right)
\end{aligned}
$$

with a constant $C$ independent of $r$ and $w$.

4. The Rankin convolution. Given two automorphic forms $f$ and $g$ we are going to construct a Dirichlet series $D(s, f, g)$ by "Rankin's method". For $(w, z) \in D$ let $d \Omega$ be the $G_{\mathbf{R}}$ invariant volume element given by

$$
d \Omega=q(w, z)^{-3} \bigwedge_{\mu \in \Delta} d w_{\mu} d \bar{w}_{\mu} d x_{\mu} d y_{\mu}
$$

(see [7, p. 574]). Here $x_{\mu}$ and $y_{\mu}$ are the real and imaginary parts of $z_{\mu}$, respectively.

Now let

$$
f(w, z)=\sum_{r \in \hat{J}} g_{r}(w) \cdot e(\operatorname{tr}(r z))
$$

and

$$
g(w, z)=\sum_{s \in \hat{J}} h_{s}(w) \cdot e(\operatorname{tr}(s z))
$$

be automorphic forms of weights $k$ and $m$, respectively. Assume $f \cdot g$ is a cusp form. Consider the integral

$$
D(s, f, g)=\left[N_{\Gamma}: P_{\Gamma}\right]^{-1} \int_{P_{\Gamma} \backslash D} f \bar{g} q(w, z)^{(k+m) / 2+s} d \Omega
$$

with $s=(s, \ldots, s)$ a complex parameter. The integrand is invariant under the action $P_{\Gamma \cdot}$ (The factor $\left[N_{\Gamma}: P_{\Gamma}\right]^{-1}$ is inserted so that $D(s, f, g)$ is independent of the choice of $P_{\Gamma}$. See $\S 2$.)

Proposition 4.1. For $\operatorname{Re}(s)$ sufficiently large

$$
\begin{aligned}
D(s, f, g)= & \frac{\operatorname{Vol}\left(\mathbf{R}^{n} / J\right)}{\left[N_{\Gamma}: P_{\Gamma}\right]}(4 \pi)^{-\lambda(s)-1} \cdot \Gamma_{n}(\lambda(s)+1) \\
& \cdot \sum_{r \in \hat{J} / U}\left\langle g_{r}, h_{r}\right\rangle r^{-\lambda(s)-1},
\end{aligned}
$$

where $\lambda(s)=\frac{1}{2}(k+m)+s-3$,

$$
\left\langle g_{r}, h_{r}\right\rangle=\int_{\mathbf{C}^{n} / L} g_{r}(w) \overline{h_{r}(w)} e(\operatorname{tr}(r \bar{w} S w)) \bigwedge_{\mu \in \Delta} d w_{\mu} d \bar{w}_{\mu},
$$


and as in the introduction,

$$
\Gamma_{n}(\lambda(s)+1)=\prod_{\mu \in \Delta} \Gamma\left(\frac{1}{2}\left(k_{\mu}+m_{\mu}\right)+s-2\right) .
$$

Proof. Assuming convergence, let us consider $D(s, f, g)$ formally at first. One sees easily that $P_{\Gamma} \backslash D$ may be represented by points $(w, x+i y)$ with

$$
w \in \mathbf{C}^{n} / U \times L, x \in \mathbf{R}^{n} / J \text {, and } y \in \mathbf{R}^{n} \text { with } y>-\frac{1}{2} \bar{w} S w .
$$

Here two points $w, w^{\prime}$ in $\mathbf{C}^{n}$ are equivalent modulo $U \times L$ if there are $u \in U$ and $v \in L$ such that $w^{\prime}=w u+v$. Integrating out the $x$-variable in (4.1) we obtain

$$
\frac{\operatorname{vol}\left(\mathbf{R}^{n} / J\right)}{\left[N_{\Gamma}: P_{\Gamma}\right]} \cdot \int_{\substack{w \in \mathbf{C}^{n} / U \times L \\ y>-\frac{1}{2} i \bar{w} S w}} \sum_{r \in \hat{J}} g_{r} \bar{h}_{r} e(2 i \cdot \operatorname{tr}(r y)) q(w, z)^{\lambda(s)} \bigwedge_{\mu \in \Delta} d w_{\mu} d \bar{w}_{\mu} d y_{\mu}
$$

Denote a typical term in the integrand by

$$
I_{r}(w, y)=\frac{\operatorname{vol}\left(\mathbf{R}^{n} / J\right)}{\left[N_{\Gamma}: P_{\Gamma}\right]} g_{r}(w) \bar{h}_{r}(w) \cdot e(2 i \cdot \operatorname{tr}(r y)) \cdot q(w, z)^{\lambda(s)} .
$$

Make the substitution $t=y+\frac{1}{2} i \bar{w} S w$ in the above integral. Also, let $u \in U$ act on $r \in \hat{J}$ by multiplication by $u^{2}$. Then the expression (4.3) may be written

$$
\int_{\substack{w \in \mathbf{C}^{n} / U \times L \\ t>0}} \sum_{r \in \hat{J} / U} \sum_{u \in U} I_{u^{2} \Gamma}\left(w, t-\frac{1}{2} i \bar{w} S w\right) \bigwedge_{\mu \in \Delta} d w_{\mu} d \bar{w}_{\mu} d t_{\mu} .
$$

Recalling formula (2.8) and substituting this in (4.4) we obtain

$$
\begin{gathered}
\frac{\operatorname{vol}\left(\mathbf{R}^{n} / J\right)}{\left[N_{\Gamma}: P_{\Gamma}\right]} \int_{\substack{w \in \mathbf{C}^{n} / L \\
t>0}} \sum_{r \in \hat{J} / U} g_{r} \bar{h}_{r} e(\operatorname{tr}(r \bar{w} S w)) t^{\lambda(s)} e^{-4 \pi \cdot \operatorname{tr}(r t)} \bigwedge_{\mu \in \Delta} d w_{\mu} d \bar{w}_{\mu} d t_{\mu} \\
=\frac{\operatorname{vol}\left(\mathbf{R}^{n} / J\right)}{\left[N_{\Gamma}: P_{\Gamma}\right]}(4 \pi)^{-\lambda(s)-1} \Gamma(\lambda(s)+1) \cdot \sum_{r \in \hat{J} / U}\left\langle g_{r}, h_{r}\right\rangle r^{-\lambda(s)-1} .
\end{gathered}
$$

To justify these manipulations we use the estimates of $\S 3$. Thus, Proposition 3.1 applied to the cusp form $f \cdot g$ implies that the integral expression (4.1) converges absolutely provided

$$
\int_{P_{\Gamma} \backslash D} q(w, z)^{-(k+m) / 2} q(w, z)^{(k+m) / 2+\operatorname{Re}(s)} d \Omega
$$

converges. That is, if $\operatorname{Re}(s)>2$. On the other hand, we can use Proposition 3.2 to estimate $\left\langle g_{r}, h_{r}\right\rangle$. Thus

$$
\begin{aligned}
\left|\left\langle g_{r}, h_{r}\right\rangle\right| & \leq \int_{\mathbf{C}^{n} / L}\left|g_{r}(w)\right| \cdot\left|h_{r}(w)\right| \cdot e(\operatorname{tr}(r \cdot \bar{w} S w))\left|\bigwedge_{\mu \in \Delta} d w_{\mu} d \bar{w}_{\mu}\right| \\
& \leq C r^{\beta}
\end{aligned}
$$


where $C$ is a positive constant independent of $r$ and

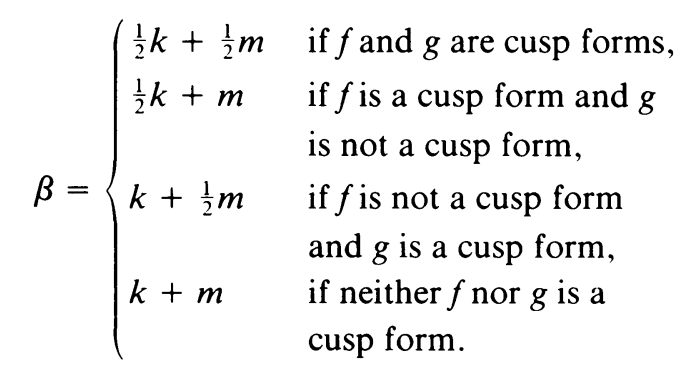

Therefore the series of (4.6) is absolutely convergent for $\operatorname{Re}(s)>3+\beta-\frac{1}{2}(k+m)$. (Note that all of the components of $\beta-\frac{1}{2}(k+m)$ are equal.) Thus, all of the previous manipulations are valid provided $\operatorname{Re}(s)>3+\beta-\frac{1}{2}(k+m)$.

We end this section by obtaining another expression for $D(s, f, g)$. Let $Q$ denote a set of representatives for $P_{\Gamma} \backslash \Gamma$. Write

$$
P_{\Gamma} \backslash D=\bigcup_{\gamma \in Q} \gamma(\Gamma \backslash D) \quad \text { (disjoint union). }
$$

Substituting this in the integral of (4.1) we obtain

$$
\begin{aligned}
& {\left[N_{\Gamma}: P_{\Gamma}\right]^{-1} \sum_{\gamma \in Q} \int_{\gamma(\Gamma \backslash D)} f \cdot \bar{g} q(w, z)^{(k+m) / 2+s} d \Omega} \\
& \quad=\left[N_{\Gamma}: P_{\Gamma}\right]^{-1} \int_{\Gamma \backslash D} \sum_{\gamma \in Q} f \cdot \gamma \overline{g \cdot \gamma} \cdot q(\gamma(w, z))^{(k+m) / 2+s} d \Omega .
\end{aligned}
$$

Using formula (3.2) this becomes

$$
\left[N_{\Gamma}: P_{\Gamma}\right]^{-1} \int_{\Gamma \backslash D} f \cdot \bar{g} E_{k-m}^{*}(z, s) \cdot q(w, z)^{(k+m) / 2+s} d \Omega,
$$

where

$$
E_{\lambda}^{*}(z, s)=\sum_{\gamma \in Q} j(\gamma, z)^{\lambda} \cdot|j(\gamma, z)|^{-\lambda-2 s} .
$$

Postponing questions of convergence, $E_{\lambda}^{*}(z, s)$ is a "group-theoretic" Eisenstein series. In a more general context Langlands [3] has studied Eisenstein series and obtained their analytic continuation and functional equation. In the next two sections we shall derive the necessary analytic information in a different, more explicit manner.

5. The coset space $P_{\Gamma} \backslash \Gamma$. To study the Eisenstein series (4.7) we need an explicit set of representatives for $P_{\Gamma} \backslash \Gamma$. Recall $(\S 2) P_{\Gamma}=Z_{\Gamma} \cdot U_{\Gamma} \cdot M(L, J)$. Let

$$
x_{\Gamma}=\{\gamma \in \Gamma \mid j(\gamma, z) \equiv 1\} \text {. }
$$

$M(L, J)$ is a subgroup of finite index in $X_{\Gamma}$. Also, any two elements of $\Gamma$ in the same right coset of $X_{\Gamma}$ have the same bottom row, and conversely. Thus the coset space $P_{\Gamma} \backslash \Gamma$ can be described naturally in terms of the bottom rows of elements of $\Gamma$, modulo a group of units. To get a clean result it is necessary to specialize 
somewhat the preceding discussion. Thus, choose $\zeta \in \mathcal{O}_{K}$ satisfying $\bar{\zeta}=-\zeta$ and $\operatorname{Im}\left(\zeta^{\mu}\right)>0$ for all $\mu \in \Delta$. Define

$$
\phi=\zeta R^{-1}=\zeta\left(\begin{array}{lll}
S^{-1} & 0 & 0 \\
0 & 0 & -1 \\
0 & 1 & 0
\end{array}\right) \quad(\operatorname{see}(1.1))
$$

We assume

$$
2 \zeta S^{-1} \in \mathcal{O}_{F} \quad\left(\text { note that } 2 \zeta S^{-1}>0\right) .
$$

Let $\phi$ define a Hermitian form on $K_{3}$ by $\phi(x, y)=x \phi^{t} \bar{y}$. Note that

$$
G_{Q}=\left\{g \in K_{3}^{3} \mid \phi(x g, y g)=\phi(x, y) \text { for all } x, y \in K_{3}\right\},
$$

i.e. $G_{Q}$ is the unitary group of the Hermitian form $\phi$. We also choose the lattice $\Lambda$ in a special form. For this, choose an arbitrary integral ideal $C$ of $\mathcal{O}_{K}$ and a fractional ideal $\mathfrak{b}$ such that $\mathcal{O}_{K} \subseteq \mathfrak{b}$ and $\mathfrak{b}^{\rho}=\mathfrak{b}$. Let

$$
\Lambda=\left\{(x, y, z) \in K_{3} \mid x \in 2 C, y \in\left(\zeta^{-1}\right), z \in \mathfrak{b}\right\} .
$$

We shall characterize the bottom rows of elements of $\Gamma=\Gamma_{\Lambda}$ for this choice of $\Lambda$.

LEMMA 5.1. The lattice $\Lambda$ has the following properties:

(1) $\Lambda$ is an $\mathcal{O}_{k}$-module,

(2) $\{\phi(x, y) \mid x, y \in \Lambda\}=\mathfrak{b}$,

(3) $\{\phi(x, x) \mid x \in \Lambda\} \subseteq \operatorname{Tr}_{K / F}(\mathfrak{b})$.

Proof. This follows easily from the definition of $\phi$ and $\Lambda$.

Write

$$
M^{\perp}=\left\{(x, 0,0) \in K_{3} \mid x \in 2 C\right\}
$$

and

$$
M=\left\{(0, y, z) \in K_{3} \mid y \in\left(\zeta^{-1}\right), z \in \mathfrak{b}\right\} .
$$

Then $\Lambda=M^{\perp} \oplus M$, the sum being orthogonal with respect to $\phi$. Call a lattice an $\mathcal{O}_{K}$-lattice if it is also an $\mathcal{O}_{K}$-module. Then $M$ is an $\mathcal{O}_{K}$-lattice in the 2-dimensional subspace $\mathbf{Q} M=\{(0, y, z) \mid y, z \in K\}$ of $K_{3}$. (Here $\mathbf{Q} M$ denotes the $\mathbf{Q}$-linear span of $M$.)

LEMMA 5.2. Let $W$ be a subspace of $K_{3}$ and $N \subseteq W$ and $\mathcal{O}_{K}$ sublattice of $\Lambda$. Suppose that there is a K-linear isomorphism $\alpha: W \rightarrow \mathbf{Q} M$ such that $\alpha(N)=M$ and $\phi(\alpha x, \alpha y)$ $=\phi(x, y)$. Then $\Lambda=N \oplus M^{\perp}$, where $N^{\perp}=\{x \in \Lambda \mid \phi(N, x)=0\}$.

Proof. If $W^{\perp}=\left\{x \in K_{3} \mid \phi(W, x)=0\right\}$, then $K_{3}=W \oplus W^{\perp}$. Since $N$ is an $\mathcal{O}_{K}$-lattice, we have $W=\mathbf{Q} N$. Therefore each element $v \in \Lambda$ may be written $v=q x$ $+y$ with $y \in W^{\perp}, q \in \mathbf{Q}$ and $x \in N$ primitive, i.e. $x / n \notin \Lambda$ for any integer $n>1$. The lemma will follow once we show $q \in Z$. Note $\phi(v, N) \subseteq \mathfrak{b}$ by Lemma 5.1 and $\phi(v, N)=q \phi(x, N)=q \phi(\alpha(x), M)$. Therefore

$$
\mathfrak{b}^{-1} \phi(\alpha(x), M)=q^{-1} I
$$


with an integral ideal $I$. Write $\alpha(x)=\left(0, \zeta^{-1} a, b\right) \in M a \in \mathcal{O}_{K}$ and $b \in \mathfrak{b}$. Then $\phi(\alpha(x), M)=a \mathfrak{b}+b \mathcal{O}_{K}$. Therefore $q^{-1} I=a \mathcal{O}_{K}+b \mathfrak{b}^{-1}$ is an integral ideal. If $q \notin$ $Z$, then the ideal $a \mathcal{O}_{K}+b \mathfrak{b}^{-1}$ has a factor of the form $n \mathcal{O}_{K}$ with an integer $n>1$. Then $n$ divides $a$ and $b / n \in=\mathfrak{b}$. This implies that $\alpha(x) / n \in M$, which contradicts the fact that $x$ is primitive.

For an element $\gamma \in K_{3}^{3}$ let $x(\gamma) \in K_{3}$ denote the bottom row of $\gamma$.

Proposition 5.3.

$$
\begin{gathered}
\left\{x(\gamma) \mid \gamma \in \Gamma_{\Lambda}\right\} \\
=\left\{x \in \mathfrak{b}^{-1} \Lambda \mid \phi(x, x)=0,1 \in \phi(x, \Lambda)\right\} .
\end{gathered}
$$

Proof. Suppose $\gamma \in G_{\mathbf{Q}}$ and $\Lambda \gamma=\Lambda$. Let $x=x(\gamma)$. The relation $\gamma \phi^{t} \bar{\gamma}=\phi$ implies $\phi(x, x)=0$. Moreover,

$$
\Lambda=\Lambda \gamma=\left(M^{\perp} \oplus M\right) \gamma .
$$

From the definition of $M(5.5)$, all elements of the form $(0,0, b)$ with $b \in \mathfrak{b}$ are in $\left(M^{\perp} \oplus M\right)$. Therefore $b \cdot x \in \Lambda$ and so $x \in \mathfrak{b}^{-1} \Lambda$. Finally, let $y$ denote the second to last row of $\gamma$. Then $x \phi^{t} \bar{y}=\zeta$. Moreover, from (5.5) it follows that $\left(0, \zeta^{-1}, 0\right) \in M^{\perp}$ $\oplus M$. Hence from (5.8), $y \in \zeta \Lambda$. Therefore $1=\phi\left(x,-\zeta^{-1} y\right) \in \phi(x, \Lambda)$. Thus (5.6) is contained in (5.7).

Conversely, suppose that $x$ is an element of (5.7). Choose $y_{0} \in \Lambda$ so that $\phi\left(x, y_{0}\right)=1$. By Lemma 5.1 there is $c \in \mathfrak{b}$ so that $\phi\left(y_{0}, y_{0}\right)=-\operatorname{tr}(c)$. Since $x \in \mathfrak{b}^{-1} \Lambda$ then $c x \in \Lambda$. Let $y=y_{0}+c x \in \Lambda$. Then

$$
\phi(x, y)=1 \quad(\text { since } \phi(x, x)=0 \text { and } \phi(y, y)=0) \text {. }
$$

Let $W$ be the $K$-subspace of $K_{3}$ generated by $x$ and $y$. The relations (5.9) show that $W$ is isometric to

$$
U=\{(0, u, v) \mid u, v \in K\}=\mathbf{Q} M
$$

through the map $\alpha_{0}: W \rightarrow U$ with $\alpha_{0}(x)=(0,0,1)$ and $\alpha_{0}(y)=\left(0, \zeta^{-1}, 0\right)$. Moreover, the map $\alpha_{0}$ takes the lattice $N=\mathcal{O}_{K} y+\mathfrak{b} x \subseteq \Lambda$ to the lattice $M$ (5.5). Therefore by Lemma 5.2, $\Lambda=N \oplus N^{\perp}$. By Witt's theorem, $\alpha_{0}$ may be extended to an element $\alpha \in G_{\mathbf{Q}}$. Then

$$
\alpha(\Lambda)=\alpha\left(N \oplus N^{\perp}\right)=M \oplus \alpha\left(N^{\perp}\right) .
$$

Moreover, since det $\alpha(\overline{\operatorname{det} \alpha})=1$ we have

$$
\gamma=\left(\begin{array}{ccc}
\operatorname{det} \alpha & & \\
& 1 & \\
0 & & \\
& & 1
\end{array}\right) \in G_{\mathbf{Q}}
$$

and so replacing $\alpha$ by $\alpha \gamma^{-1}$ we may suppose that det $\alpha=1$. Note $\alpha\left(N^{\perp}\right)=$ $\{(x, 0,0) \mid x \in I\}$ for some ideal $I \subseteq K$. Write $J=2 C$ (see 5.3). Recall $M=$ $\left\{(0, y, z) \mid y \in\left(\zeta^{-1}\right), z \in \mathfrak{b}\right\}$. Write

$$
\alpha^{-1}=\left(\begin{array}{lll}
a_{1} & b_{1} & c_{1} \\
a_{2} & b_{2} & c_{2} \\
a_{3} & b_{3} & c_{3}
\end{array}\right) .
$$


From (5.10) it follows that $I a_{1} \subseteq J, I b_{1} \subseteq\left(\zeta^{-1}\right), I c_{1} \subseteq \mathfrak{b}, \zeta^{-1} a_{2} \in J$, etc. Then a simple computation shows $\operatorname{det}\left(\alpha^{-1}\right) \in I^{-1} J$. But $\operatorname{det}(\alpha)=1$, so $I \cdot J^{-1}$ is integral. Similarly $I^{-1} J$ is integral and so $I=J$, proving that $\alpha(\Lambda)=\Lambda$. Now $x\left(\alpha^{-1}\right)=x$, proving that (5.7) is contained in (5.6). (Note that as a matrix $\alpha^{-1}$ acts on the right.)

REMARK. A natural choice for a discrete subgroup of $G_{\mathbf{Q}}$ is $G_{\mathbf{Q}} \cap \mathrm{SL}_{3}\left(\mathcal{O}_{K}\right)$. To see how Proposition 5.3 applies in this case, choose $C=\mathcal{O}_{K}$ and $\mathfrak{b}=\left(\zeta^{-1}\right)$ in (5.3). Let

$$
\sigma=\left(\begin{array}{ccc}
2 \zeta & & 0 \\
& 1 & \\
0 & & 1
\end{array}\right)
$$

Note that $\sigma G_{\mathbf{Q}} \sigma^{-1}$ is the unitary group of $\phi(5.1)$ with $S^{-1}$ replaced by $4 \zeta \bar{\zeta} S^{-1}$ and that $\sigma \Gamma_{\Lambda} \sigma^{-1}=\left(\sigma G_{\mathbf{Q}} \sigma^{-1}\right) \cap \mathrm{SL}_{3}\left(\mathcal{O}_{K}\right)$. Moreover, $(x, y, z)$ is the bottom row of an element of $\sigma \Gamma_{\Lambda} \sigma^{-1}$ if and only if $(2 \zeta, x, y, z)$ is the bottom row of an element of $\Gamma_{\Lambda}$.

6. Eisenstein series. Let $\phi$ be as in (5.1). For elements $z=(w, z) \in D$ and $x=(a, b, c) \in K_{3}$, let $x \cdot z=a w+b z+c$. For two $\mathcal{O}_{K}$-lattices $\Lambda_{1}, \Lambda_{2} \subseteq K_{3}$ such that $\phi\left(\Lambda_{1}, \Lambda_{2}\right) \subseteq \mathcal{O}_{K}$ define

$$
E_{\lambda}^{*}\left(z, s ; \Lambda_{1}, \Lambda_{2}\right)=\sum_{x \in \mathscr{R} / \mathcal{O}_{F}^{*}}(x \cdot z)^{\lambda} \cdot|x \cdot z|^{-2 s-\lambda},
$$

where $\mathscr{R}=\left\{x \in \Lambda_{1}-\{0\} \mid \phi(x, x)=0, \phi\left(x, \Lambda_{2}\right)=\mathcal{O}_{K}\right\}, 0 \leq \lambda=\left(\lambda_{\mu}\right)_{\mu \in \Delta}$ is an $n$-tuple of integers, and $s \in \mathbf{C}$. The series is well defined provided $u^{\lambda} \cdot|u|^{-\lambda}=1$ for every $u \in \mathcal{O}_{F}^{x}$. Assume this condition. When we have occasion to vary $\Lambda_{1}$ we will write $\mathscr{R}=\mathscr{R}\left(\Lambda_{1}\right)$.

THEOREM 6.1. The series (6.1) is convergent for $\operatorname{Re}(s)$ sufficiently large and defines an analytic function of $s$. Suppose $\lambda_{\mu}>0$ for some $\mu \in \Delta$. Then the function $E_{\lambda}^{*}\left(\mathrm{z}, s ; \Lambda_{1}, \Lambda_{2}\right)$ can be analytically continued to a meromorphic function on the whole $s$-plane. Moreover, when multiplied by a suitable product of $L$-functions and $\Gamma$-factors it becomes an entire function.

Proof. The proof of this theorem will be rather long. To begin, let us ignore questions of convergence and consider the series of (6.1) formally at first. Let $\iota$ denote the Möbius function on the integral ideals of $\mathcal{O}_{K}$ and $C$ a set of integral representatives for the ideal class group of $K$. Let $\mathscr{R}_{I}=\mathscr{R}_{I}\left(\Lambda_{1}\right)=\left\{x \in \Lambda_{1}-\right.$ $\left.\{0\} \mid \phi(x, x)=0, \phi\left(x, \Lambda_{2}\right) \subseteq I\right\}$. Letting $I$ run over all integral ideals, we have

$$
\begin{aligned}
E_{\lambda}^{*}\left(z, s ; \Lambda_{1}, \Lambda_{2}\right) & =\sum_{I} \iota(I) \cdot \sum_{x \in \mathscr{R}_{I} / \mathcal{O}_{F}^{x}}(x \cdot z)^{\lambda} \cdot|x \cdot z|^{-2 s-\lambda} \\
& =\sum_{A \in C} \sum_{\beta \in A^{-1} / \mathcal{O}_{K}^{x}} \iota(\beta A) \sum_{x \in \mathscr{R}_{\beta A} / \mathcal{O}_{F}^{x}}(x \cdot z)^{\lambda}|x \cdot z|^{-2 s-\lambda} .
\end{aligned}
$$

Writing $y=\beta^{-1} x$ this last sum becomes

$$
\sum_{A \in C} \sum_{\beta \in A^{-1} / \mathcal{O}_{K}^{x}} \iota(\beta A) \cdot \beta^{\lambda} \cdot|\beta|^{-2 s-\lambda} \sum_{y \in \mathscr{R}_{A}\left(\beta^{-1} \Lambda_{1}\right) / \mathcal{O}_{F}^{x}}(y \cdot z)^{\lambda} \cdot|y \cdot z|^{-2 s-\lambda} .
$$


Letting $\hat{\Lambda}_{2}=\left\{x \in K_{3} \mid \phi\left(x, \Lambda_{2}\right) \subseteq \mathcal{O}_{K}\right\}$, we have by assumption $\Lambda_{1} \subseteq \hat{\Lambda}_{2}$. If $y \in K_{3}$ satisfies $\phi\left(y, \Lambda_{2}\right) \subseteq A$, then $y \in A \hat{\Lambda}_{2}$. Thus the inner sum of (6.2) may be restricted to the lattice $\beta^{-1} \Lambda_{1} \cap \mathrm{A} \hat{\Lambda}_{2}$. Note that $\beta \in A^{-1}$ so $A \Lambda_{1} \subseteq \beta^{-1} \Lambda_{1}$. Thus

$$
A \Lambda_{1} \subseteq \beta^{-1} \Lambda_{1} \cap A \hat{\Lambda}_{2} \subseteq A \hat{\Lambda}_{2} .
$$

As $\left[A \hat{\Lambda}_{2}: A \Lambda_{2}\right]<\infty$ it follows that once $A$ is chosen there are only finitely many possibilities for the lattice $\beta^{-1} \Lambda_{1} \cap A \hat{\Lambda}_{2}$. Denoting these lattices by $L_{A},(6.2)$ may be written

$$
\sum_{A \in C} \sum_{\Lambda \in L_{A}} \sum_{\beta} \iota(\beta A) \cdot \beta^{\lambda} \cdot|\beta|^{-2 s-\lambda} \sum_{y \in \mathscr{R}_{A}(\Lambda) / \mathcal{O}_{F}^{x}}(y \cdot z)^{\lambda} \cdot|y \cdot z|^{-2 s-\lambda},
$$

where $\beta$ is summed over elements $\beta \in A^{-1} / \mathcal{O}_{F}^{x}$ such that $\beta^{-1} \Lambda_{1} \cap A \hat{\Lambda}_{2}=\Lambda$. Since $C$ and $L_{A}$ are finite sets, the analytic continuation of the series $E_{\lambda}^{*}\left(z, s ; \Lambda_{1}, \Lambda_{2}\right)$ is reduced to the continuation of the following two types of series:

$$
\sum_{\beta} \iota(\beta A) \cdot \beta^{\lambda} \cdot|\beta|^{-2 s-\lambda}
$$

and

$$
\sum_{y \in \mathscr{R}_{A}(\Lambda) / \mathcal{O}_{F}^{x}}(y \cdot z)^{\lambda}|y \cdot z|^{-2 s-\lambda} .
$$

The series (6.3) has an analytic continuation by virtue of the fact that it has a simple expression in terms of Hecke $L$-functions. The reader interested in the details should consult $\left[8\right.$, p. 659], where a similar problem is treated. (The condition $\beta^{-1} \Lambda_{1} \cap A \hat{\Lambda}_{2}$ $=\Lambda$ represents finitely many congruence conditions on $\beta$.)

Let us now consider series of type (6.4). Since $\mathscr{R}_{A}(\Lambda)=\left\{x \in \Lambda \cap A \hat{\Lambda}_{2} \mid x \neq 0\right.$, $\phi(x, x)=0\}$, it is enough to consider series of the form

$$
E_{\lambda}(z, s ; \Lambda)=\sum_{m \in R(\Lambda) / \mathcal{O}_{F}^{x}}(m \cdot z)^{\lambda}|m \cdot z|^{-2 s-\lambda},
$$

where $\Lambda \subseteq \hat{\Lambda}_{2}$ is an arbitrary $\mathcal{O}_{K}$-lattice and $R(\Lambda)=\{x \in \Lambda \mid x \neq 0, \phi(x, x)=0\}$. Our method of analytic continuation is essentially that of $[9, \S 13]$, where the case of orthogonal groups is considered. We include a detailed treatment here so as to be able to obtain certain necessary estimates. For a point $z=(w, z) \in D$ let

$$
Q(z)=\left(\begin{array}{ccc}
S^{-1} & 0 & w \\
\bar{w} & \bar{z} & z \\
0 & 1 & 1
\end{array}\right)
$$

By a simple computation (see [7, p. 572])

$$
\phi=Q(z) \cdot\left(\begin{array}{cc}
\xi(z)^{-1} & 0 \\
0 & -\eta(z)^{-1}
\end{array}\right) \cdot{ }^{t} \bar{Q}(z),
$$

where

$$
\xi(z)^{-1}=\zeta \cdot\left(\begin{array}{cc}
-S^{-1} & w \\
-\bar{w} & z-\bar{z}
\end{array}\right)^{-1}
$$


and

$$
\eta(z)^{-1}=-\zeta(\bar{w} S w+\bar{z}-z)^{-1} .
$$

Since $\eta(z)>0$ and $\phi$ has signature $(2,1)$, we conclude that $\xi(z)>0$. Let

$$
\mathscr{P}(z)=Q(z) \cdot\left(\begin{array}{cc}
\xi(z)^{-1} & 0 \\
0 & +\eta(z)^{-1}
\end{array}\right) \cdot{ }^{t} \bar{Q}(z) .
$$

Then $\mathscr{P}(z)>0 .\left(\mathscr{P}(z)\right.$ is a minimal majorant of $\phi$.) If $m \in K_{3}$ satisfies $\phi(m, m)=0$, then from (6.6), (6.7) and (6.10) we have

$$
\left(m \mathscr{P}(z)^{t} \bar{m}\right)^{s+\lambda / 2}=\left(2 \eta(z)^{-1}\right)^{s+\lambda / 2}|m \cdot z|^{2 s+\lambda} .
$$

With this equality in mind, our goal is to rewrite the series $E_{\lambda}(z, s ; \Lambda)$ as a sort of "Epstein zeta function". To be more precise let $\delta \in K$ be chosen such that $\operatorname{Im} \delta^{\sigma}>0$ for all $\sigma \in \Delta$ and $\delta^{2}=-d \in F$ with $d$ totally positive. Fixing $\delta$ once and for all, for any matrix $A$ with coefficients in $K$ define $R(A)=\frac{1}{2}\left(A+A^{\rho}\right)$ and $I(A)=$ $\frac{1}{2} \delta^{-1}\left(A-A^{\rho}\right)$. Now define maps from $K_{3}^{3}$ to $F_{6}^{6}$ which map $A \in K_{3}^{3}$ to

$$
\tilde{A}=\left(\begin{array}{cc}
R(A) & I(A) \\
-I(A) & d^{-1} R(A)
\end{array}\right)
$$

and $m \in K_{3}$ to $\tilde{m}=(R(m), d I(m))$. Under this map a lattice $\Lambda \subseteq K_{3}$ becomes a lattice $\tilde{\Lambda} \subseteq F_{6}$. Using the isomorphism $K \otimes_{\mathbf{Q}} \mathbf{R}=\mathrm{C}_{n}$ (via the $C M$-type $\Delta$ ) we may extend these maps to $\mathbf{R}$-linear maps from $\left(\mathbf{C}_{3}^{3}\right)_{n}$ to $\left(\mathbf{R}_{6}^{6}\right)_{n}$ and $\left(\mathbf{C}_{3}\right)_{n}$ to $\left(\mathbf{R}_{6}\right)_{n}$. Then it is easily verified that $\operatorname{Re}\left(v A^{t} \bar{w}\right)=\tilde{v} \tilde{A}^{t} \tilde{w}$ for $v, w \in\left(\mathbf{C}_{3}\right)_{n}$ and $A \in\left(\mathbf{C}_{3}^{3}\right)_{n}$. Let us write $\varphi=\tilde{\phi}$ and $P=P(z)=\overline{\mathscr{P}(z)}$. We need the following lemma.

LEMMA 6.2. Let $r=r(z)=\left(0,0,-2,0,0,-2 \delta^{-1}\right) \cdot \overline{Q(z)}^{-1}$. Then

(1) $\varphi \cdot{ }^{t} r=-P \cdot{ }^{t} r$

(2) $r \varphi \cdot{ }^{t} r=0$,

(3) If $m \in K_{3}$, then $\left(r \varphi \cdot{ }^{t} \tilde{m}\right)^{\lambda}=\left(2 \eta(z)^{-1}(m \cdot z)\right)^{\lambda}$.

This lemma may be proved by a straightforward calculation.

Combining Lemma 6.2 with (6.11) we have

$$
E_{\lambda}(z, s ; \Lambda)=\sum_{m \in R(\tilde{\Lambda}) / \mathcal{O}_{F}^{x}}\left(\frac{1}{2} \eta(z)\right)^{-s+\lambda / 2}\left(r \varphi^{t} m\right)^{\lambda} \cdot\left(m P^{t} m\right)^{-s-\lambda / 2}
$$

Here

$$
R(\tilde{\Lambda})=\left\{m \in \tilde{\Lambda} \mid m \neq 0, m \varphi^{t} m=0\right\} .
$$

Since $P>0$ it is a standard fact that the right-hand side of (6.12) converges for $\operatorname{Re}(s)$ sufficiently large. Let $X$ be a coset of a lattice in $F_{6}$. We generalize the series (6.12) by defining

$$
G_{\lambda}(z, s ; X)=\sum_{m \in R(X) / U_{X}}\left(r \varphi^{t} m\right)^{\lambda}\left(m P^{t} m\right)^{-s-\lambda / 2} .
$$

Here $U_{X} \subseteq \mathcal{O}_{F}^{x}$ is the group of units which stabilize the coset $X$. We shall now obtain the analytic continuation of this series. 
For a variable $\tau=u+i v \in \mathfrak{h}^{n}$ ( $\mathfrak{h}$ is the upper half plane) define a (nonholomorphic) theta series by

$$
\theta_{\lambda}(\tau, \xi ; x)=\sum_{m \in X}\left(r \varphi^{t} m\right)^{\lambda} \cdot e\left(\operatorname{tr}\left(u \cdot m \varphi^{t} m\right)+i \cdot \operatorname{tr}\left(v \cdot m P^{t} m\right)\right) .
$$

Let $T(\varphi, z)$ denote the $\mathrm{C}$-vector space generated by functions of the form

$$
f(\tau ; z, a, h, Y)=\sum_{m \in Y}\left(r \varphi^{t} m\right)^{\lambda} e\left[a\left(\operatorname{tr}\left(u \cdot m \varphi^{t} m+i v m P^{t} m\right)\right)+m \cdot h\right],
$$

with arbitrary $0 \ll a \in F, h \in F^{6}$, and $Y$ a coset of a lattice in $F_{6}$. For a function $g(\tau)$ on $\mathfrak{h}^{n}$ and an element $\gamma=\left(\begin{array}{ll}a & b \\ c & d\end{array}\right) \in \mathrm{GL}_{2}(F)$ with totally positive determinant define

$$
g \mid \gamma(\tau)=g(\gamma(\tau)) \cdot(c \tau+d)^{-2} \cdot(c \bar{\tau}+d)^{-1-\lambda} .
$$

PROPOSITION 6.3. There is a congruence subgroup $\Upsilon$ of $\mathrm{SL}_{2}\left(\mathcal{O}_{F}\right)$ so that

$$
\theta_{\lambda}(\tau, \jmath ; X) \mid \gamma=\theta_{\lambda}(\tau, \jmath ; X)
$$

for all $\gamma \in \Upsilon$. Moreover, given $\gamma \in \mathrm{GL}_{2}(F)$ with totally positive determinant,

$$
\theta_{\lambda}(\tau, z ; X) \mid \gamma \in T(\varphi, z) \text {, }
$$

and the expression of this function as an element of $T(\varphi, z)$ is independent of $z$.

For the proof of this proposition we refer the reader to [9, Proposition 7.1].

To continue with the proof of Theorem 6.1, there is a lattice $L \subseteq \mathcal{O}_{F}$ and a subgroup $U \subseteq \mathcal{O}_{F}^{x}$ of finite index so that

$$
\Upsilon_{\infty} \stackrel{\text { def }}{\equiv}\left\{\left(\begin{array}{ll}
1 & b \\
0 & 1
\end{array}\right) \in \Upsilon\right\}=\left\{\left(\begin{array}{ll}
1 & b \\
0 & 1
\end{array}\right) \mid b \in L\right\}
$$

and

$$
U_{\Upsilon} \stackrel{\text { def }}{\equiv}\left\{\left(\begin{array}{cc}
u & 0 \\
0 & u^{-1}
\end{array}\right) \in \Upsilon \mid u>0\right\}=\left\{\left(\begin{array}{cc}
u & 0 \\
0 & u^{-1}
\end{array}\right) \mid u \in U\right\} .
$$

Consider the integral

$$
\int_{U_{\Upsilon} \Upsilon_{\infty} \backslash \mathfrak{h}^{n}} \boldsymbol{\theta}_{\lambda}(\tau, z ; X) v^{s+\lambda / 2} \frac{d u d v}{v},
$$

observing that the integrand is invariant under $U_{\Upsilon} \Upsilon_{\infty}$. A fundamental domain for $U_{\Upsilon} \Upsilon_{\infty}$ is $\mathbf{R}^{n} / L+i \cdot\left(U^{2} \backslash \mathbf{R}_{+}^{n}\right)$, where $L$ acts on $\mathbf{R}^{n}$ by translation and $U^{2}$ acts on $\mathbf{R}_{+}^{n}=\{v \in \mathbf{R} \mid v>0\}^{n}$ by multiplication. Integrating out the $u$-variable in (6.18) we obtain

$$
\operatorname{Vol}\left(\mathbf{R}^{n} / L\right) \cdot \int_{U^{2} \backslash \mathbf{R}_{+}^{n}} \sum_{m \in R(X)}\left(r \varphi^{t} m\right)^{\lambda} \cdot e\left(i \operatorname{tr}\left(v \cdot m P^{t} m\right)\right) v^{s+\lambda / 2} \frac{d v}{v} .
$$

(Note that by definition (6.13), $0 \notin R(X)$. This causes no problem above as we have assumed $\lambda_{\mu}>0$ for some $\mu \in \Delta$.) Using a well-known principle we may rewrite (6.19) as

$$
\operatorname{Vol}\left(\mathbf{R}^{n} / L\right) \int_{\mathbf{R}_{+}^{n}} \sum_{m \in R(X) / U}\left(r \varphi^{t} m\right)^{\lambda} \cdot e\left(i \operatorname{tr}\left(v \cdot m P^{t} m\right)\right) v^{s+\lambda / 2} \frac{d v}{v}
$$




$$
\begin{gathered}
=\operatorname{Vol}\left(\mathbf{R}^{n} / L\right) \Gamma\left(s+\frac{1}{2} \lambda\right)(2 \pi)^{-s-\lambda / 2} \sum_{m \in R(X) / U}\left(r \varphi^{t} m\right)^{\lambda}\left(m P^{t} m\right)^{-s-\lambda / 2} \\
=\operatorname{Vol}\left(\mathbf{R}^{n} / L\right) \Gamma\left(s+\frac{1}{2} \lambda\right)(2 \pi)^{-s-\lambda / 2}\left[U_{X}: U\right] G_{\lambda}(z, s ; X)
\end{gathered}
$$

(see (6.14)). All of the above steps are justified for $\operatorname{Re}(s)$ sufficiently large since the series of (6.21) is absolutely convergent in that range. Thus we obtain the series $G_{\lambda}(z, s ; X)$ in terms of the integral of (6.18). To obtain the analytic continuation of $G_{\lambda}(z, s ; X)$ write

$$
U_{\Upsilon} \Upsilon_{\infty} \backslash \mathfrak{h}^{n}=\bigcup_{\gamma \in R} \gamma\left(\Upsilon \backslash \mathfrak{h}^{n}\right),
$$

where $R$ is a set of representatives for $\{ \pm 1\} U_{\Upsilon} \Upsilon_{\infty} \backslash\{ \pm 1\} \Upsilon$. Substituting this in (6.18) we obtain

$$
\int_{\Upsilon \backslash \mathfrak{h}^{n}} \theta_{\lambda}(\tau, \mathfrak{z} ; X) H_{\lambda}^{*}(t, s) \frac{d u d v}{v^{2}}
$$

where

$$
H_{\lambda}^{*}(\tau, s)=v^{s+1} \sum_{\left(c_{d}^{*}\right) \in R}(c \tau+d)^{2}(c \bar{\tau}+d)^{1+\lambda}|c \tau+d|^{-2 s-2-\lambda} .
$$

The series $H_{\lambda}^{*}(\tau, s)$ is an Eisenstein series on the Hilbert modular group, and hence its analytic properties are well known. From [8, Proposition 3.2] it follows that there is a function $T(s)$, which is a product of $L$-functions and $\Gamma$-factors, so that $H_{\lambda}(\tau, s) \equiv T(s) \cdot H_{\lambda}^{*}(\tau, s)$ possesses an analytic continuation to an entire function on the whole $s$-plane. Moreover, given $\gamma \in \mathrm{GL}(2, F)$ with totally positive determinant, and a compact set $S \subseteq \mathbf{C}$, there is a constant $A>0$ (depending on $\gamma$ and $S$ ) so that

$$
H_{\lambda}(\tau, s) \mid \gamma=O\left(v_{1} \cdots v_{n}\right)^{A} \quad \text { as }\left(v_{1}, \ldots, v_{n}\right) \rightarrow(\infty, \ldots, \infty),
$$

the estimate being uniform in $s \in S$. To complete the analytic continuation of $G_{\lambda}(z, s ; X)$ it remains to show that the integral (6.23) converges for all $s \in \mathbf{C}$ (at least when it is multiplied by $T(s)$ ). For this we first review the reduction theory for the Hilbert modular group. Thus, there is a finite set $P \subseteq \operatorname{SL}(2, F)$ and a set

$$
W(\delta, \varepsilon) \equiv\left\{u+i v \in \mathfrak{h}^{n}|| u \mid<\delta, v>\varepsilon\right\}
$$

with positive constants $\delta$ and $\varepsilon$, such that

$$
\mathfrak{h}^{n}=\Upsilon \cdot \bigcup_{\gamma \in P} \gamma(W(\delta, \varepsilon)) \text {. }
$$

Therefore

$$
\begin{aligned}
& \left|\int_{\Upsilon \backslash \mathfrak{h}{ }^{n}} \theta_{\lambda}(\tau, z ; X) H_{\lambda}(t, s) \frac{d u d v}{v^{2}}\right| \\
& \quad \leq \int_{W(\delta, \varepsilon)} \sum_{\gamma \in P}\left|\theta_{\lambda}\right|(t, z ; X)|\gamma| \cdot\left|H_{\lambda}\right|(t, s)|\gamma| \frac{d u d v}{v^{2}} .
\end{aligned}
$$

Since $\lambda_{\mu}>0$ for some $\mu \in \Delta$ all the series $\theta_{\lambda}(\tau, z ; X)$ are zero at the cusp $i \infty$. Hence the estimate (6.24) shows that the above integral converges uniformly on compact 
subsets $S \subseteq \mathbf{C}$. This proves that $T(s) \cdot G_{\lambda}(z, s ; X)$ is entire and completes the proof of Theorem 6.1.

For the proof of our main theorem we will need an estimate in the $z$-variable of the function $E_{\lambda}^{*}\left(z, s ; \Lambda_{1}, \Lambda_{2}\right)$ (see (6.1)).

Proposition 6.4. Let $S$ be a compact subset of $\mathbf{C}$. Then there exist positive constants $A, B$, and $C$ depending on $S$, and a polynomial $R(w, \bar{w}, z, \bar{z})$ independent of $S$, so that

$$
\left|T(s) E_{\lambda}^{*}\left(z, s ; \Lambda_{1}, \Lambda_{2}\right)\right| \leq C \cdot R(w, \bar{w}, z, \bar{z})^{A} \cdot q(w, z)^{-B}
$$

for all $s \in S, z \in D .\left(\right.$ Recall $q(w, z)=\operatorname{Im}(z)+\frac{1}{2} i^{t} \bar{w} S w$. $)$

For the proof of Proposition 6.4 we will need two lemmas.

LeMMA 6.5. If $r$ and $\varepsilon$ are positive constants and $A \in \mathbf{R}$, then

$$
\int_{v^{\prime}>\varepsilon} e^{-\left(r v_{1} \cdots v_{n}\right)}\left(v_{1} \ldots v_{n}\right)^{A} d v_{1} \cdots d v_{n} \leq C \cdot r^{-B}
$$

where $C$ and $B$ are positive constants depending only on $\varepsilon$ and $A$. (The integration is performed over the region $v_{1} \geq \varepsilon, \ldots, v_{n} \geq \varepsilon$.)

We omit the proof of this lemma, which is routine.

LEMMA 6.6. Let $B$ be a nonnegative number and $r=\left(r_{\mu}\right)_{\mu \in \Delta}$ with $0<r_{\mu} \leq 1$. Then

$$
\sum_{0<m \in \mathcal{O}_{F}} m^{B} e^{-\operatorname{tr}(r \cdot m)} \leq C \cdot r^{-B-1}
$$

with a constant $C>0$ depending on $B$ and $\mathcal{O}_{F}$ but not on $r$.

Proof. Let $a_{1}, \ldots, a_{n}$ be a $\mathbf{Z}$-basis of $\mathcal{O}_{F}$ consisting of totally positive elements $a_{i}$. Let $\mathcal{O}_{F}$ be embedded in $\mathbf{R}^{n}$ via $\Delta$. If $0<m \in \mathcal{O}_{F}$, then the parallelepiped $P(m)$ spanned by the line segments $\overline{m, m+a_{1}}, \ldots, \overline{m, m+a_{n}}$ is entirely contained in $\mathbf{R}_{+}^{n}$. Moreover, if $x \in P(m)$, then

$$
\begin{aligned}
x^{B} e^{-\operatorname{tr}(r \cdot x)} & \geq m^{B} e^{-\operatorname{tr}\left(r \cdot\left(m+a_{1}+\cdots+a_{n}\right)\right)} \\
& \geq m^{B} e^{-\operatorname{tr}\left(a_{1}+\cdots+a_{n}\right)} e^{-\operatorname{tr}(r \cdot m)},
\end{aligned}
$$

since $0<r \leq 1$. Therefore

$$
\sum_{0<m \in \mathcal{O}_{F}} m^{B} e^{-\operatorname{tr}(r \cdot m)} \leq C \cdot \sum_{0<m \in \mathcal{O}_{F}} \int_{P(m)} x^{B} e^{-\operatorname{tr}(r \cdot x)} d x_{1} \cdots d x_{n}
$$

with a constant $C$ depending on $\mathcal{O}_{F}$. The right-hand side of the above inequality is majorized by

$$
C \int_{\mathbf{R}^{n}} x^{B} e^{-\operatorname{tr}(r \cdot x)} d x_{1} \cdots d x_{n}=C \cdot \Gamma(B+1)^{n} r^{-B-1} .
$$

Proof of Proposition 6.4. For convenience we recall a formula obtained in the proof of Theorem 6.1. (Note that from the proof of Theorem 6.1 it follows that it is sufficient to estimate the series $G_{\lambda}(\mathrm{z}, s ; X)(6.14)$.)

$$
T(s) G_{\lambda}(\mathfrak{z}, s ; X)=\frac{(2 \pi)^{s+\lambda / 2} \Gamma(s+\lambda / 2)^{-1}}{\left[U_{X}: U\right] \operatorname{Vol}\left(\mathbf{R}^{n} / L\right)} \int_{\Upsilon \backslash \mathfrak{h}^{n}} \theta_{\lambda}(\tau, \jmath ; \quad X) H_{\lambda}(t, s) \frac{d u d v}{v^{2}}
$$


(see (6.18)-(6.23).) Therefore we can estimate $T(s) G_{\lambda}(z, s, X)$ by the inequality (6.26). For this we need to estimate $\theta_{\lambda}(\tau, z ; X) \mid \gamma$ in $z$. By Proposition 6.3 it is enough to estimate any element $f(\tau, z)=f(\tau ; z, a, h, Y)$ of $T(\varphi, z)$ (see (6.16)). Thus, let $\alpha=\alpha(z)$ and $\beta=\beta(z)$ be the minimal and maximal eigenvalues of $P$, respectively. Since $r \varphi=-r P$ and $\left|r P^{t} m\right| \leq\left|r P^{t} r\right|^{1 / 2} \cdot\left|m P^{t} m\right|^{1 / 2}$ we have

$$
\begin{aligned}
|f(\tau, z)| & \leq C_{1} \sum_{m \in\left(\mathcal{O}_{F}\right)_{6}}\left|r P^{t} r\right|^{\lambda / 2}\left|m P^{t} m\right|^{\lambda / 2} e\left(i a \operatorname{tr}\left(v m P^{t} m\right)\right) \\
& \leq C_{1}\left|r P^{t} r\right|^{\lambda / 2} \beta^{\lambda / 2} \sum_{m \in\left(\mathcal{O}_{F}\right)_{6}}\|m\|^{\lambda} e\left(i a \cdot \operatorname{tr}\left(v \alpha\|m\|^{2}\right)\right),
\end{aligned}
$$

where $C_{1}$ is a positive constant independent of $z$, and $\|m\|^{2}=m_{1}^{2}+\cdots+m_{6}^{2}$ if $m=\left(m_{1}, \ldots, m_{6}\right) \in F_{6}$. For $0<m \in \mathcal{O}_{F}$ let $g_{6}(m)=\#\left\{x \in\left(\mathcal{O}_{F}\right)_{6} \mid\|x\|^{2}=m\right\}$. One sees easily that $g_{6}(m) \leq \mathrm{Cm}^{3}$ with a constant $C$ independent of $m$. Thus from (6.32) we obtain

$$
|f(\tau, z)| \leq C_{2}\left|r P^{t} r\right|^{\lambda / 2} \beta^{\lambda / 2} \sum_{0<m \in \mathcal{O}_{F}} m^{\lambda / 2+3} e(i a \cdot \operatorname{tr}(v \alpha m)) .
$$

Now, for $v>\varepsilon>0$ and $0<m \in \mathcal{O}_{F}$,

$$
\operatorname{tr}(v \cdot \alpha m) \geq \varepsilon \operatorname{tr}(\alpha \cdot m)
$$

and

$$
\operatorname{tr}(v \cdot \alpha m) \geq n(v \alpha m)^{1 / n} \geq n \alpha^{1 / n} \cdot v^{1 / n} .
$$

(The first inequality of line (6.35) follows from the arithmetic mean-geometric mean inequality.) Write

$$
e(i a \cdot \operatorname{tr}(v \alpha m))=e\left(\frac{1}{2} i a \cdot \operatorname{tr}(v \alpha m)\right) \cdot e\left(\frac{1}{2} i a \cdot \operatorname{tr}(v \alpha m)\right)
$$

and apply (6.34) to the first factor and (6.35) to the second factor. Then for $v>\varepsilon$ we obtain from (6.33)

$$
|f(\tau, z)| \leq C_{2}\left|r P^{t} r\right|^{\lambda / 2} \beta^{\lambda / 2} e\left(\frac{1}{2} i n a^{1 / n} \cdot v^{1 / n}\right) \sum_{0<m \in \mathcal{O}_{F}} m^{B} e\left(\frac{1}{2} \text { iastr }(\alpha \cdot m)\right)
$$

with $B=\frac{1}{2} \lambda+3$. Applying Lemmas 6.5 and 6.6 and the estimate (6.24) we obtain (for $s \in S$ )

$$
\left.\int_{W(\delta, \varepsilon)}|f(\tau, z)| \cdot\left|H_{\lambda}(t, s)\right| \gamma\left|\frac{d u d v}{v^{2}} \leq C_{3}\right| r P^{t} r\right|^{\lambda / 2} \beta^{\lambda / 2} \alpha^{-B_{1}}\left(\alpha^{\prime}\right)^{-B_{2}}
$$

where $\alpha^{\prime}=\min (1, a \pi \varepsilon \alpha)$ and $C_{3}, B_{1}$, and $B_{2}$ are positive constants independent of $z$. Now, for any positive definite Hermitian matrix $H$ with eigenvalues $0<\delta_{1} \leq \ldots \leq$ $\delta_{m}$ we have $\delta_{1}+\cdots+\delta_{m}=\operatorname{tr} H$ and $\delta_{1} \cdots \delta_{m}=\operatorname{det} H$. Therefore $\delta_{m} \leq \operatorname{tr} H$ and $\delta_{1} \geq \operatorname{det}(H) \cdot \operatorname{tr}(H)^{1-m}$. Thus the right-hand side of (6.37) is majorized by

$$
C_{3}\left|r P^{t} r\right|^{\frac{1}{2} \lambda}(\operatorname{tr} P)^{\frac{1}{2} \lambda} \frac{(\operatorname{tr} P)^{5 B_{1}}}{(\operatorname{det} P)^{B_{1}}} \min \left(1, \frac{a \pi \varepsilon \operatorname{det} P}{(\operatorname{tr} P)^{5}}\right)^{-B_{2}} \text {. }
$$


From the formulas for $P$ and $r$ (see (6.10) and Lemma 6.2) we see that (6.38) is majorized by an expression of the form

$$
C \cdot R(w, \bar{w}, z, \bar{z})^{A} \cdot q(w, z)^{-B}
$$

with a polynomial $R$ and positive constants $A, B$, and $C$ independent of $z=(w, z)$. Combining this with (6.31) and (6.26) concludes the proof of Proposition 6.4.

7. Analytic continuation of Dirichlet series. We now combine the results of the previous sections to obtain our main theorem. Choose $\phi$ and $\Lambda$ as in $\S 5$ (see (5.1) and (5.3)). Let $\Gamma=\Gamma_{\Lambda}=\left\{\gamma \in G_{\mathbf{Q}} \mid \Lambda \gamma=\Lambda\right.$, $\left.\operatorname{det}(\gamma)=1\right\}$. Now suppose $f$ and $g$ are automorphic forms with respect to $\Gamma$, of weights $k$ and $m$, respectively. Recall the definition of $D(s, f, g)$ from $\S 4,(4.1)$.

TheOREM 7.1. Assume $f \cdot g$ is a cusp form. Suppose $k \geq m, k_{\mu}>m_{\mu}$ for some $\mu \in \Delta$, and $u^{k-m} \cdot|u|^{m-k}=1$ for all $u \in \mathcal{O}_{F}^{x}$. Then the Dirichlet series $D(s, f, g)$ possesses an analytic continuation to the whole s-plane. Moreover, when multiplied by a suitable product of L-functions and $\Gamma$-factors it becomes an entire function.

Proof. As in (4.11) we may write

$$
D(s, f, g)=\frac{1}{\left[N_{\Gamma}: P_{\Gamma}\right]} \int_{\Gamma \backslash D} f \bar{g} E_{k-m}^{*}(z, s ; \Gamma) q(z)^{(k+m) / 2+s} d \Omega
$$

Here

$$
E_{k-m}^{*}(\jmath, s ; \Gamma)=\sum_{\gamma \in Q} j(\gamma, \jmath)^{k-m}|j(\gamma, z)|^{-2 s+m-k}
$$

with $Q$ a set of representatives for $P_{\Gamma} \backslash \Gamma$.

Now, by Proposition 5.3 there is a natural correspondence between the coset space $P_{\Gamma} \backslash \Gamma$ and the set $\left\{x \in \mathfrak{b}^{-1} \Lambda \mid \phi(x, x)=0,1 \in \phi(x, \Lambda)\right\} / Z U$, with $Z$ a finite group of roots of unity and $U \subseteq \mathcal{O}_{F}^{x}$ a subgroup of finite index. The correspondence is $\left[X_{\Gamma}: M(L, J)\right]$ to one (see (5.0)). Hence we obtain from (7.2) (with $C=$ $\left.\left[\mathcal{O}_{F}^{x}: U\right] /[Z: 1] \cdot\left[X_{\Gamma}: M(L, J)\right]\right)$.

$$
E_{k-m}^{*}(z, s ; \Gamma)=c E_{k-m}^{*}\left(z, s ; \mathfrak{b}^{-1} \Lambda, \Lambda\right) .
$$

Here $E_{k-m}^{*}\left(\mathfrak{z}, s ; \mathfrak{b}^{-1} \Lambda, \Lambda\right)$ is defined by (6.1). By Theorem 6.1 there is a product $T(s)$ of $L$-functions and $\Gamma$-factors so that $T(s) E_{k-m}^{*}\left(\mathfrak{z}, s ; \mathfrak{b}^{-1} \Lambda, \Lambda\right)$ is an entire function. Therefore the series

$$
\begin{aligned}
& {\left[N_{\Gamma}: P_{\Gamma}\right] \cdot T(s) \cdot D(s, f, g)} \\
& \left.\quad=c \cdot \int_{\Gamma \backslash D} f \bar{g} T(s) E_{k-m}^{*}\left(\mathfrak{z}, s ; \mathfrak{b}^{-1} \Lambda, \Lambda\right) q(z)\right)^{(k+m) / 2+s} d \Omega
\end{aligned}
$$

will be an entire function provided that the integral converges for all $s \in \mathbf{C}$. That is does is a consequence of the reduction theory and the estimate (6.27) of Proposition 
6.4. Thus, write $D=\Gamma \cdot \bigcup_{\gamma \in P} \gamma T^{\prime}(\varepsilon, A, B)$ as in the reduction theory of $\S 3$. Then the integral in (7.4) is bounded in absolute value by

$$
\begin{aligned}
& \int_{\cup_{\gamma \in P} \gamma T(\varepsilon, A, B)}|f g| \cdot\left|T(s) E_{k-m}^{*}\left(z, s ; \mathfrak{b}^{-1} \Lambda, \Lambda\right)\right| q(\mathfrak{z})^{(k+m) / 2+\operatorname{Re}(s)}|d \Omega| \\
& =\sum_{\gamma \in P} \int_{T(\varepsilon, A, B)}|f \circ \gamma \cdot g \circ \gamma|\left|T(s) E_{k-m}^{*}\left(\gamma(z), s ; \mathfrak{b}^{-1} \Lambda, \Lambda\right)\right| \\
& \quad \leq\left.\sum_{\gamma \in P} \int_{T(\varepsilon, A, B)}|f|_{k} \gamma \cdot g\right|_{m} \gamma|\cdot| T(s) E_{k-m}^{*}\left(z, s ; \mathfrak{b}^{-1} \Lambda \gamma, \Lambda \gamma\right)\left|q(z)^{(k+m) / 2+\operatorname{Re}(s)}\right| d \Omega \mid .
\end{aligned}
$$

Now, $\left.\left.f\right|_{k} \gamma \cdot g\right|_{m} \gamma$ is a cusp form, and this together with the estimate (6.27) shows that the above integral converges uniformly for $s$ in a compact subset of $\mathbf{C}$. (As this fact may not be obvious we sketch a proof here. From Proposition 3.2 we may estimate $\left.\left.f\right|_{k} \gamma \cdot g\right|_{m} \gamma$ by an expression of the form $\sum r^{(k+m) / 2} e^{-2 \pi \cdot \operatorname{tr}(r \cdot q(z))}$, where the sum is over totally positive elements $r$ of a lattice in $F$. As in $\S 6$, we may split the exponential into two pieces and use the arithmetic mean-geometric mean inequality on the first piece and Lemma 6.6 on the second piece. Then for $z \in T(\varepsilon, A, B)$,

$$
\left.|f|_{k} \gamma \cdot g\right|_{m} \gamma \mid \leq C \cdot e^{-D q(z)^{1 / n}}
$$

where $C$ and $D$ are positive constants independent of $z \in T(\varepsilon, A, B)$. From this, Lemma 6.5, and (6.27) the convergence of the integral follows readily.)

8. A special case. We illustrate Theorem 7.1 by considering a special case in more detail. Let $K=\mathbf{Q}(i), \Delta=$ identity , and

$$
\phi=\left(\begin{array}{ccc}
\frac{1}{2} & 0 & 0 \\
0 & 0 & -i \\
0 & i & 0
\end{array}\right)
$$

Then $D=\left\{\left.(w, z) \in \mathbf{C}^{2}|\operatorname{Im}(z)>| w\right|^{2}\right\}$. Choose the lattice $\Lambda$ to be $\mathbf{Z}[i]_{3}$. Then $\Gamma=\Gamma_{\Lambda}=G_{\mathbf{Q}} \cap \operatorname{SL}(3, \mathbf{Z}[i])$. This is not a special case of the type of lattice considered in $\S 5$, but we do have a one-to-one correspondence between the bottom rows of elements of $\Gamma$ and $\left\{x \in \mathbf{Z}[i]_{3} \mid x \phi^{t} \bar{x}=0,1 \in \phi(x, \Lambda)\right\}$ (see Proposition 5.3). To consider Eisenstein series, note that in this case

$$
P_{\Gamma}=\left\{\left(\begin{array}{ccc}
1 & 0 & c \\
2 i c & 1 & i \cdot|c|^{2}+d \\
0 & 0 & 1
\end{array}\right) \mid c \in \mathbf{Z}[i], d \in \mathbf{Z}\right\} .
$$

For an integer $\lambda>0$ let

$$
E_{\lambda}^{*}(z, s)=\sum_{\gamma \in P_{\Gamma} \backslash \Gamma} j(\gamma, z)^{\lambda}|j(\gamma, z)|^{-2 s} .
$$

(We are using a slight variation of the notation employed in $\S 6.) E_{\lambda}^{*}(z, s)$ is zero unless $\lambda$ is divisible by 4 , which we now assume. For an ideal $I$ of $Q(i)$ let $\alpha_{I}$ denote a generator of $I$. Define

$$
L_{\lambda}(s)=\sum_{I} \alpha_{I}^{\lambda}\left|\alpha_{I}\right|^{-2 s}
$$


From the description of the bottom rows of $\Gamma$ we see easily that

$$
E_{\lambda}(z, s) \stackrel{\text { def }}{=} L_{\lambda}(s) E_{\lambda}^{*}(z, s)=\sum_{\substack{0 \neq x \in \mathbf{Z}[i]_{3} \\ x \phi^{\prime} \bar{x}=0}}(x \cdot z)^{\lambda}|x \cdot z|^{-2 s} .
$$

For $\tau=u+i v \in \mathfrak{h}$ define a (nonholomorphic) theta series

$$
\theta_{\lambda}(z, \tau)=\sum_{m \in \mathbf{Z}_{6}}\left(r \varphi^{t} m\right)^{\lambda} e\left(-2 u m \varphi^{t} m+2 i v m P^{t} m\right)
$$

(see $\$ 6$ for the notation). Then

$$
\begin{aligned}
\int_{v^{\prime}=0}^{\infty} & \int_{u=0}^{1} \theta_{\lambda}(z, \tau) v^{s-1} d u d v \\
& =(4 \pi)^{-s} \Gamma(s)\left(y-|w|^{2}\right)^{s-\lambda} E_{\lambda}(z, s) .
\end{aligned}
$$

Moreover, a detailed analysis shows

$$
\theta_{\lambda}(z, \gamma(\tau))=(-1)^{(d-1) / 2}(c \tau+d)^{\lambda+1}(c \bar{\tau}+d)^{2} \theta_{\lambda}(z, t)
$$

for $\gamma=\left(\begin{array}{ll}a & b \\ c & d\end{array}\right) \in \Gamma_{0}(8) \subseteq \operatorname{SL}(2, \mathbf{Z})$. (To prove this we use the method of Shimura, [6, §2].) We can then express the integral in (8.1) in terms of an integral over $\Gamma_{0}(8) \backslash \mathfrak{h}$. This introduces a (classical) Eisenstein series into the integrand. As before, this series admits an analytic continuation, and thus implies the analytic continuability of $E_{\lambda}(\jmath, s)$. However, it also admits a functional equation of a manageable sort (see [6, Lemma 3.3]). This can be used to obtain a functional equation for $E_{\lambda}(z, s)$. The calculations are long and tedious, depending on explicit transformation laws for theta functions. We shall not reproduce them here, although we will state the final result: Let $\chi$ be the Dirichlet character of $(\mathbf{Z} / 4 \mathbf{Z})^{x}$ given by $\chi(d)=(-1)^{(d-1) / 2}$. Let

$$
L(\chi, s)=\sum_{d=1}^{\infty} \chi(d) \cdot d^{-s}
$$

and write $F_{\lambda}(z, s)=T_{\lambda}(s)\left(y-|w|^{2}\right)^{s+1-\lambda} E_{\lambda}(z, s+1)$, where

$$
T_{\lambda}(s)=4^{2 s} \pi^{-s} \Gamma(s)(4 \pi)^{-s-1} \Gamma(s+1) L(\chi, 2 s+1-\lambda) .
$$

Then

$$
F_{\lambda}(z, \lambda-s)=-F_{\lambda}(z, s) .
$$

We now consider the Rankin convolution in this special case. An automorphic form $f$ of weight $k$ with respect to $\Gamma$ has a Fourier-Jacobi expansion of the form

$$
f(w, z)=\sum_{r=0}^{\infty} g_{r}(w) \cdot e(r z) .
$$

One can show that $\Gamma \backslash D$ has only one cusp, so $f$ is a cusp form if and only if $g_{0}(w)=0$ in the above expansion. Let $g$ be an automorphic form of weight $m$ with respect to $\Gamma$. Supposing that $f \cdot g$ is a cusp form, we define as before

$$
D(s, f, g)=\frac{1}{4} \int_{P_{\Gamma} \backslash D} f \bar{g}\left(y-|w|^{2}\right)^{(k+m) / 2+s} d \Omega
$$


(see (4.1)). In this case $d \Omega=\left(y-|w|^{2}\right)^{-3} d x d y d w d \bar{w}$. Let us suppose $k>m$ and that $k-m$ is divisible by 4 . Applying the technique of $\S 4$ to the integral in (8.3) we obtain

$$
D(s, f, g)=\frac{1}{4} \int_{\Gamma \backslash D} f \bar{g}\left(y-|w|^{2}\right)^{(k+m) / 2+s} E_{k-m}^{*}\left(z, s+\frac{1}{2}(k-m)\right) d \Omega .
$$

As we have just seen, $E_{\lambda}(z, s)=L_{\lambda}(s) E_{\lambda}^{*}(z, s)$ possesses an analytic continuation and functional equation (8.2). Applying this to $D(s, f, g)$, let

$$
\mathscr{D}(s)=T_{k-m}(s+(k-m) / 2-1) L_{k-m}(s+(k-m) / 2) D(s, f, g) .
$$

Then we have obtained the following proposition:

Proposition 8.1. $\mathscr{D}(s)$ is an entire function of $s$ and satisfies the functional equation

$$
\mathscr{D}(2-s)=-\mathscr{D}(s) \text {. }
$$

To understand the meaning of the $\Gamma$-factors and $L$-factors in $\mathscr{D}(s)$ we return to the paper of Shintani [10]. In the introduction to that paper the results are stated for the special case of the unitary group given above. Thus if $f$ is an automorphic form which is a simultaneous eigenfunction of the Hecke operators and $\zeta(s, f)$ is the corresponding Euler product, then $\zeta(s, f)$ multiplied by a $\Gamma$-factor is essentially given as a product of a Dirichlet $L$-function, a Hecke $L$-function of the field $\mathbf{Q}(i)$, and $D\left(s, f, g_{f}\right)$. Here $g_{f}$ is an automorphic form dependent on $f$. The two $L$-factors correspond to the two $L$-factors appearing in $\mathscr{D}(s)$ above. Thus our result indicates that the function $\zeta(s, f)$ multiplied by three $\Gamma$-factors becomes an entire function with a functional equation. (Note that the Euler factors of $\zeta(s, f)$ are of degree six in the rational primes.) In connection with this discussion we mention the papers [11, 12, and 13].

9. Congruence subgroups. In this section we briefly indicate how to generalize the preceding results to congruence subgroups of $\Gamma$. We shall make a number of simplifying assumptions: Let $\Lambda=\left(\mathcal{O}_{k}\right)_{3}$ and $\Gamma=G_{\mathbf{Q}} \cap \operatorname{SL}\left(3, \mathcal{O}_{K}\right)$ (see the remark at the end of $\$ 5)$. Write

$$
\phi=\zeta \cdot\left(\begin{array}{ccc}
t & 0 & 0 \\
0 & 0 & -1 \\
0 & 1 & 0
\end{array}\right)
$$

and assume $t \in \mathcal{O}_{k}$. We do not lose much generality by these assumptions, and anyway, the general case may be handled in much the same manner. By a congruence subgroup of $\Gamma$ we mean a subgroup containing $\{\gamma \in \Gamma \mid(\gamma-1) \Lambda \subseteq N$. $\Lambda$ \} for some integer $N$. Write

$$
\gamma=\left(\begin{array}{lll}
a_{1} & b_{1} & c_{1} \\
a_{2} & b_{2} & c_{2} \\
a_{3} & b_{3} & c_{3}
\end{array}\right)
$$


and define subgroups

$$
\begin{gathered}
\Gamma(N)=\left\{\gamma \in \Gamma \mid a_{1} \equiv b_{2} \equiv c_{2} \equiv 1 \bmod N, c_{1} \equiv a_{3} \equiv b_{1} \equiv a_{2} \equiv 0 \bmod N,\right. \\
\left.\quad \text { and } b_{3} \equiv c_{2} \equiv 0 \bmod N^{2}\right\}, \\
\Gamma_{1}(N)=\left\{\gamma \in \Gamma \mid a_{1} \equiv b_{2} \equiv c_{3} \equiv 1 \bmod N, b_{1} \equiv a_{3} \equiv 0 \bmod N,\right. \\
\text { and } \left.b_{3} \equiv 0 \bmod N^{2}\right\},
\end{gathered}
$$

and

$$
\Gamma_{0}(N)=\left\{\gamma \in \Gamma \mid a_{3} \equiv b_{1} \equiv 0 \bmod N, b_{3} \equiv 0 \bmod N^{2}\right\}
$$

Clearly $\Gamma(N) \subseteq \Gamma_{1}(N) \subseteq \Gamma_{0}(N)$, and a subgroup of $\Gamma$ is a congruence subgroup if and only if it contains $\Gamma(N)$ for some $N$.

LEMMA 9.1. If $f(w, z)$ is an automorphic form of weight $k$ with respect to $\Gamma(N)$, then $f\left(N w, N^{2} z\right)$ is an automorphic form of weight $k$ with respect to $\Gamma_{1}\left(N^{2}\right)$.

Proof. Let

$$
\sigma=\left(\begin{array}{ccc}
N & 0 & 0 \\
0 & N^{2} & 0 \\
0 & 0 & 1
\end{array}\right) .
$$

By a simple computation $\Gamma_{1}\left(N^{2}\right) \subseteq \sigma^{-1} \Gamma(N) \sigma$. But $f\left(N w, N^{2} z\right)=\left.f\right|_{k} \sigma$ is clearly an automorphic form of weight $k$ with respect to $\sigma^{-1} \Gamma(N) \sigma$.

Write $S_{k}(N)$ for the space of all automorphic forms of weight $k$ with respect to $\Gamma_{1}(N)$. Define a homomorphism $\pi: \Gamma_{0}(N) \rightarrow\left(\mathcal{O}_{K} / N\right)^{x}$ by $\pi(\gamma)=c_{3}$, with $\gamma$ written as in (9.1). For a character $\chi$ of $\left(\mathcal{O}_{K} / N\right)^{x}$ and an $n$-tuple of nonnegative integers $k$, denote by $S_{k}(N, \chi)$ the space of all holomorphic function $f: D \rightarrow \mathbf{C}$ satisfying

$$
f(\gamma(z))=\chi(\pi(\gamma)) \cdot j(\gamma, z)^{k} \cdot f(z)
$$

for all $\gamma \in \Gamma_{0}(N)$.

LEMMA 9.2. (1) $T_{1}(N)=\left\{\gamma \in \Gamma \mid a_{3} \equiv 0 \bmod N, b_{3} \equiv 0 \bmod N^{2}, c_{3} \equiv 1 \bmod N\right\}$,

(2) $\Gamma_{0}(N)=\left\{\gamma \in \Gamma \mid a_{3} \equiv 0 \bmod N, b_{3} \equiv 0 \bmod N^{2}\right\}$,

(3) $\Gamma_{1}(N)=\operatorname{ker}(\pi)$, and

(4) $S_{k}(N)=\oplus_{\chi} S_{k}(N, \chi)$.

Proof. With $\gamma \in \Gamma$ written as in (8.1), the equation $\gamma \phi^{t} \gamma^{\rho}=\phi$ implies

$$
\left(\begin{array}{lll}
a_{1} & b_{1} & c_{1} \\
a_{2} & b_{2} & c_{2}
\end{array}\right) \cdot\left(\begin{array}{ccc}
t & 0 & 0 \\
0 & 0 & -1 \\
0 & 1 & 0
\end{array}\right) \cdot\left(\begin{array}{c}
a_{3}^{\rho} \\
b_{3}^{\rho} \\
c_{3}^{\rho}
\end{array}\right)=\left(\begin{array}{c}
0 \\
-1
\end{array}\right)
$$

or

$$
t a_{1} a_{3}^{\rho}+c_{1} b_{3}^{\rho}-b_{1} c_{3}^{\rho}=0
$$

and

$$
t a_{2} a_{3}^{\rho}+c_{2} b_{3}^{\rho}-b_{2} c_{3}^{\rho}=-1 \text {. }
$$

Suppose $a_{3} \equiv 0 \bmod N$ and $b_{3} \equiv 0 \bmod N^{2}$. Then equation (9.2) shows $b_{1} \equiv 0 \bmod$ $N$, since $c_{3}$ is prime to $N$. Suppose also $c_{3} \equiv 1 \bmod N$. Then equation (8.3) implies 
$b_{2} \equiv 1 \bmod N$. As $\operatorname{det}(\gamma)=1$, it follows that $a_{1} \equiv 1 \bmod N$ as well. This proves assertions (1) and (2), and statements (3) and (4) follow easily from these.

Given $f \in S_{k}(N, \chi)$ and $g \in S_{m}(N, \psi)$ the Dirichlet series $D(s, f, g)$ is defined as before (see (4.1)). Using Proposition 5.3 one sees easily that the Eisenstein series arising in the integrand is substantially the same as the Eisenstein series considered in $\S 6$. Thus it can be analytically continued and once again we obtain an analytic continuation of $D(s, f, g)$.

Note. The sense of Lemma 9.1 is that the Dirichlet series arising from automorphic forms on congruence subgroups are no more general than those coming from automorphic forms on $\Gamma_{1}(N)$. Part (4) of Lemma 9.2 shows that we may even restrict our attention to the spaces $S_{k}(N, \chi)$. This is in close analogy with the case of elliptic modular forms on congruence subgroups of $\operatorname{SL}(2, \mathbf{Z})$.

10. The case of general unitary groups. In this concluding section we make some brief remarks on the case of unitary groups of more general signature than previously considered. In this case we take $G_{\mathbf{Q}}$ as the unitary group of the matrix

$$
R=\left(\begin{array}{ccc}
S & 0 & 0 \\
0 & 0 & 1_{p} \\
0 & -1_{p} & 0
\end{array}\right)
$$

Here $S \in \mathrm{GL}(q, K)$ satisfies ${ }^{t} S^{\rho}=-S$ and $-i S^{\mu}>0$ for each $\mu \in \Delta$. Thus the real points of $G_{\mathbf{Q}}$ can be identified with $n$ copies of a unitary group of signature $(p+q, p)$. This groups acts on a Hermitian symmetric domain $D=\prod_{\mu \in \Delta} D_{\mu}$ analogous to (1.3). (For more details see [7].) The material in $\S \S 1,2,3$ and 4 can be duplicated in this more general setting. However, Proposition 5.3 of $\$ 5$ does not generalize as stated. To state a theorem in the general case denote by $x(g)$ the bottom $p$ rows of an element $g \in \mathrm{GL}(2 p+q, K)$. Define an element $x \in\left(\mathcal{O}_{K}\right) \sum_{p+q}$ to be primitive if $x=x(g)$ for some $g \in \mathrm{GL}\left(2 p+q, \mathcal{O}_{K}\right)$. Let

$$
\Gamma=G_{\mathbf{Q}} \cap \operatorname{SL}\left(2 p+q, \mathcal{O}_{K}\right) \text {. }
$$

Then we have

Proposition 10.1. There exist finitely many elements $\alpha_{1}, \ldots, \alpha_{m}$ of $G_{\mathbf{Q}}$ so that

$$
\begin{aligned}
\{x & \left.\in\left(\mathcal{O}_{K}\right)_{2 p+q}^{p} \mid x \phi^{t} \bar{x}=0, x \text { is primitive }\right\} \\
& =\bigcup_{i=1}^{m}\left\{a x\left(\alpha_{i} \gamma\right) \mid a \in \operatorname{GL}\left(p, \mathcal{O}_{K}\right), \gamma \in \Gamma\right\} .
\end{aligned}
$$

Moreover, the union (10.2) is disjoint.

The proposition asserts that under left multiplication by $\operatorname{GL}\left(p, \mathcal{O}_{K}\right)$ and right multiplication by $\Gamma$, the set (10.1) has finitely many double cosets. This follows from the well-known finiteness of the double coset space $N_{\mathbf{Q}} \backslash G_{\mathbf{Q}} / \Gamma$, with $N_{\mathbf{Q}}$ defined as in (2.2). Proposition 5.3 essentially asserts that in the case $p=q=1$, we may take $m=1$. Without proof we state that this continues to hold whenever $q=0$ or 1 , and that in general $m>1$ if $q>1$. 
In $\S 6$ we obtained the analytic continuation of Eisenstein series defined as sums of a factor of automorphy over a set such as (10.1) (see (6.1)). This method seems only to work in the case $p=1$ (rank one unitary groups). Restricting to this case but allowing $q>1$ we can generalize Theorem 7.1. Unfortunately, we do not obtain the analytic continuation of $D(s, f, g)$, but rather of $\sum_{i=1}^{m} c_{i} D\left(s, f_{i}, g_{i}\right)$, where $f_{i}=f \circ \alpha_{i}^{-1}$ and $g_{i}=g \circ \alpha_{i}^{-1}$, with $\alpha_{1}, \ldots, \alpha_{m}$ as in Proposition 10.1, and the $c_{i}$ are certain numerical constants independent of $f, g$ and $s$. This unsatisfying situation is imposed on us by Proposition 10.1, and is the reason we restricted to the case $p=q=1$ throughout the rest of this paper. It would be interesting to refine Proposition 10.1 by obtaining an explicit characterization of those elements $x \in\left(\mathcal{O}_{K}\right) \sum_{p+q}$ which are the bottom $p$ rows of an element of $\Gamma$. As already stated the conditions $x \phi^{t} \bar{x}=0$ and $x$ primitive are not in general sufficient. One may hope to remedy this by imposing some congruence conditions on $x$ and $\Gamma$, but this will not work in general.

NoTE ADDED IN PROOF. The author has recently learned of some recent work of Piatetski-Shapiro and Rallis in which they obtain new integral representations and analytic continuations of some $L$-functions attached to classical groups, including unitary groups.

\section{REFERENCES}

1. A. Borel, Reduction theory for arithmetic groups, Algebraic Groups and Discontinuous Subgroups, Sympos. Pure Math., vol. 9, Amer. Math. Soc., Providence, R. I., 1966.

2. Y. Flicker, L-packets and liftings for $U(3)$, preprint.

3. R. P. Langlands, On the functional equations satisfied by Eisenstein series, Lecture Notes in Math., vol. 544, Springer, Berlin and New York, 1976.

4. O. T. O’Meara, Introduction to quadratic forms, Springer-Verlag, Berlin and New York, 1971.

5. G. Shimura, Arithmetic of unitary groups, Ann. of Math. 79 (1964), 369-409.

6. __ On modular forms of half integral weight, Ann. of Math. 97 (1973), 440-481.

7. The arithmetic of automorphic forms with respect to a unitary group, Ann. of Math. 107 (1978), 569-605.

8. The special values of zeta functions associated with Hilhert modular forms, Duke Math. J. 45 (1978), 637-679.

9. The arithmetic of certain zeta functions and automorphic forms on orthogonal groups, Ann. of Math. 111 (1980), 313-375.

10. T. Shintani, On automorphic forms on unitary groups of order 3, preprint.

11. S. Kudla, On certain Euler products for $S U(2,1)$, Compositio Math. 42 (1981), 321-344.

12. S. Gelbart and I. I. Piatetski-Shapiro, Automorphic forms and L-functions for the unitary group, preprint 1983.

13. I. I. Piatetski-Shapiro, Tate theory for reductive groups and distinguished representations, Proc. Internat. Congress Math. (Helsinki, 1978), Acad. Sci. Fenn, 1980, pp. 585-590.

School of Mathematics, Institute for Advanced Study, Princeton, New Jersey 08540

Current address: Department of Mathematics. Yale University, New Haven, Connecticut 06520 